\title{
MAPPINGS OF FINITE DISTORTION BETWEEN METRIC MEASURE SPACES
}

\author{
CHANG-YU GUO
}

\begin{abstract}
We establish the basic analytic properties of mappings of finite distortion between proper Ahlfors regular metric measure spaces that support a $(1,1)$-Poincaré inequality. As applications, we prove that under certain integrability assumption for the distortion function, the branch set of a mapping of finite distortion between generalized $n$-manifolds of type $A$ has zero Hausdorff $n$-measure.
\end{abstract}

\section{INTRODUCTION}

Let $\Omega \subset \mathbb{R}^{n}$ be a domain. A mapping $f: \Omega \rightarrow \mathbb{R}^{n}$ is said to be a mapping of finite distortion if $f \in W_{l o c}^{1,1}\left(\Omega, \mathbb{R}^{n}\right), J_{f} \in L_{\text {loc }}^{1}(\Omega)$ and if there is a measurable function $K: \Omega \rightarrow[1, \infty]$ such that

$$
|D f(x)|^{n} \leq K(x) J_{f}(x) \text { a.e. in } \Omega,
$$

where $|D f(x)|$ and $J_{f}(x)$ are the operator norm and the Jacobian determinant of the matrix $D f(x)$, respectively. Associated with such a mapping the (outer) distortion $K(\cdot, f)$ is defined as

$$
K(x, f)=\frac{|D f(x)|^{n}}{J_{f}(x)}, \quad \text { if } J_{f}(x)>0,
$$

and $K(x, f)=1$ otherwise. When $K(\cdot, f)$ is (essentially) bounded, we recover the well-known class of quasiregular mappings or mappings with bounded distortion. The study of quasiregular mappings was initiated with Reshetnyak [33, and the basic theory was comprehensively laid and significantly advanced in a sequence of papers from the Finnish school of Martio, Rickman and Väisälä in the late 1960s [29 31, 34]. The study of mappings of finite distortion was (partially) motivated by nonlinear elasticity theory of Antman, Ball and Ciarlet [21] and the whole theory is well developed now; see [18, 20, 23, 27].

In recent years, there has been growing interest to study geometric function theory beyond the Euclidean spaces, especially in the setting of nonsmooth metric measure spaces; see [10, 12, 14, 16, 25, 32, 42, 44. The purpose of this paper is to study mappings of finite distortion between nonsmooth metric measure spaces.

It was perhaps Heinonen and Koskela [12] who first systematically developed the basic theory of quasiconformal maps, that is, quasiregular homeomorphisms, between general metric spaces with controlled geometry. This theory also initiated

Received by the editors October 24, 2014.

2010 Mathematics Subject Classification. Primary 30C65; Secondary 30L99, 57P99.

The author was partially supported by the Academy of Finland grant 131477 and the Magnus Ehrnrooth foundation. 
a new way of looking at weakly differentiable maps between nonsmooth spaces. It was somehow surprising that such a rich theory exists merely assuming that the underlying measure is doubling and an abstract Poincaré inequality holds. Later, Cheeger systematically investigated the structure of doubling metric measure spaces with the abstract Poincaré inequality in 4 and proved that actually a strong measurable differentiable structure exists for such spaces. In 14, the Sobolev class of Banach space valued mappings was studied and several characterizations of quasiconformal mappings between Ahlfors regular Loewner spaces were established. Following the general Sobolev space theory [14,15, Marshall Williams recently studied the equivalence of different definitions for quasiregular mappings between metric measure spaces of locally bounded geometry; see [44].

Of particular interest for us is the theory of a subclass of quasiregular maps, called mappings of bounded length distortion (BLD), between non-smooth spaces, developed by Heinonen and Rickman [16. The motivation there was to use BLDmaps to study problems in geometric topology. Heinonen and Sullivan [17] applied the theory to study a widely open problem: characterize metric spaces that are locally bi-Lipschitz homeomorphic to some Euclidean spaces $\mathbb{R}^{n}$. The non-smooth setting, in both studies, is the so-called generalized manifolds of type A; see Section 5 and Section 6 below for precise definitions. Several interesting questions arose from [16], for example, it was implicitly asked whether a general theory of quasiregular maps between generalized manifolds of type A exists. Very recently, Onninen and Rajala [32] partially solved the problem. To be more precise, they developed a basic theory of quasiregular maps from Euclidean domains to generalized manifolds with restricted topology and locally controlled geometry (which are more general than generalized manifolds of type $A$ ). More recently, Kirsilä 25] has established the basic properties of mappings of finite distortion from generalized manifolds with controlled geometry to Euclidean spaces.

These are the motivations of the current paper and the paper is then naturally divided into two parts. In the first part, we develop analytic properties of mappings of finite distortion between proper Ahlfors $n$-regular metric measure spaces that support a $(1,1)$-Poincaré inequality. In fact, most results hold under weaker assumption, i.e. the space supports a $(1, n)$-Poincaré inequality. We mainly investigate the following properties of mappings of finite distortion (possibly with some assumptions for the distortion function): Condition $N$, area formula, condition $N^{-1}$, Cheeger's differentiability, and $\mathscr{H}^{n}$-a.e. positiveness of the Jacobian. In the second part, we consider mappings of finite distortion between generalized manifolds of type $A$. We have shown that under suitable integrability condition for the distortion function, the branch set of a mapping of finite distortion $f$ between generalized manifolds of type $A$ has zero Hausdorff $n$-measure.

We have benefited much from the paper [16] considering the BLD mappings between generalized manifold of type $A$, and from the forthcoming monograph [15] considering the general Newtonian-Sobolev space theory on metric measure spaces.

\section{Sobolev Spaces and Poincaré inequalities on metric measure spaces}

In this section, we will briefly introduce the Sobolev spaces on metric measure spaces based on an upper gradient approach. For a detailed description of this approach, see the monograph [15]. 


\subsection{Metric measure spaces.}

Definition 2.1. A metric measure space is defined to be a triple $(X, d, \mu)$, where $(X, d)$ is a separable metric space and $\mu$ is a nontrivial locally finite Borel regular measure on $X$.

Definition 2.2. A Borel regular measure $\mu$ on a metric space $(X, d)$ is called a doubling measure if every ball in $X$ has positive and finite measure and there exists a constant $C_{\mu} \geq 1$ such that

$$
\mu(B(x, 2 r)) \leq C_{\mu} \mu(B(x, r))
$$

for each $x \in X$ and $r>0$. We call the triple $(X, d, \mu)$ a doubling metric measure space if $\mu$ is a doubling measure on $X$. We call $(X, d, \mu)$ an Ahlfors $n$-regular space, $1 \leq n<\infty$, if there exists a constant $C \geq 1$ such that

$$
C^{-1} r^{n} \leq \mu(B(x, r)) \leq C r^{n}
$$

for all balls $B(x, r) \subset X$ of radius $r<\operatorname{diam} X$.

It is well known that if $(X, d, \mu)$ is an Ahlfors $n$-regular space, then

$$
\mu(E) \approx \mathscr{H}^{n}(E)
$$

for all Borel sets $E$ in $X$; see [9, Chapter 8]. In this regard, it will be convenient to use the Hausdorff $n$-measure for an Ahlfors $n$-regular metric spaces.

2.2. Modulus of a curve family. Let $(X, d)$ be a metric space. A curve in $X$ is a continuous map $\gamma: I \rightarrow X$, where $I \subset \mathbb{R}$ is an interval. We call $\gamma$ compact, open, or half-open, depending on the type of the interval $I$.

Given a compact curve $\gamma:[a, b] \rightarrow X$, its length is the supremum of the sums

$$
\sum_{i=1}^{k} d\left(\gamma\left(t_{i}\right), \gamma\left(t_{i-1}\right)\right),
$$

where the numbers $t_{i}$ run over all finite sequences of points of the form

$$
a=t_{0}<t_{1}<\cdots<t_{k}=b .
$$

If $\gamma$ is not compact, its length is defined to be the supremum of the lengths of the compact subcurves of $\gamma$. Thus, every curve has a well-defined length in the extended nonnegative reals, and we denote it by length $(\gamma)$ or simply $l(\gamma)$.

A curve is said to be rectifiable if its length $l(\gamma)$ is finite, and locally rectifiable if each of its compact subcurves is rectifiable. For any rectifiable curve $\gamma$ there are its associated length function $s_{\gamma}: I \rightarrow[0, l(\gamma)]$ and a unique 1-Lipschitz map $\gamma_{s}$ : $[0, l(\gamma)] \rightarrow X$ such that $\gamma=\gamma_{s} \circ s_{\gamma}$. The curve $\gamma_{s}$ is the arc length parametrization of $\gamma$.

When $\gamma$ is rectifiable, and parametrized by arclength on the interval $[a, b]$, the integral of a Borel function $\rho: X \rightarrow[0, \infty]$ along $\gamma$ is

$$
\int_{\gamma} \rho d s=\int_{0}^{l(\gamma)} \rho\left(\gamma_{s}(t)\right) d t .
$$

Similarly, the line integral of a Borel function $\rho: X \rightarrow[0, \infty]$ over a locally rectifiable curve $\gamma$ is defined to be the supremum of the integral of $\rho$ over all compact subcurves of $\gamma$. 
Let $X=(X, d, \mu)$ be a metric measure space as defined in 2.1). Let $\Gamma$ be a family of curves in $X$. A Borel function $\rho: X \rightarrow[0, \infty]$ is admissible for $\Gamma$ if for every locally rectifiable curve $\gamma \in \Gamma$,

$$
\int_{\gamma} \rho d s \geq 1
$$

The $p$-modulus of $\Gamma, p \geq 1$, is defined as

Observe that

$$
\operatorname{Mod}_{p}(\Gamma)=\inf \left\{\int_{X} \rho^{p} d \mu: \rho \text { is admissible for } \Gamma\right\} .
$$

$$
\begin{gathered}
\operatorname{Mod}_{p}(\emptyset)=0 \\
\operatorname{Mod}_{p}\left(\Gamma_{1}\right) \leq \operatorname{Mod}_{p}\left(\Gamma_{2}\right), \text { if } \Gamma_{1} \subset \Gamma_{2},
\end{gathered}
$$

and

$$
\operatorname{Mod}_{p}\left(\bigcup_{i=1}^{\infty} \Gamma_{i}\right) \leq \sum_{i=1}^{\infty} \operatorname{Mod}_{p}\left(\Gamma_{i}\right) .
$$

Moreover, if $\Gamma_{0}$ and $\Gamma$ are two curve families such that each curve $\gamma \in \Gamma$ has a subcurve $\gamma_{0} \in \Gamma_{0}$, then

$$
\operatorname{Mod}_{p}(\Gamma) \leq \operatorname{Mod}_{p}\left(\Gamma_{0}\right) .
$$

We say in such a situation that $\Gamma$ majorizes $\Gamma_{0}$.

2.3. Sobolev spaces based on upper gradients. Let $X=(X, d, \mu)$ be a metric measure space and $Z=\left(Z, d_{Z}\right)$ a metric space.

Definition 2.3. A Borel function $g: X \rightarrow[0, \infty]$ is called an upper gradient for a map $f: X \rightarrow Z$ if for every rectifiable curve $\gamma:[a, b] \rightarrow X$, we have the inequality

$$
\int_{\gamma} g d s \geq d_{Z}(f(\gamma(b)), f(\gamma(a)))
$$

If inequality (2.10) merely holds for $p$-almost every compact curve, then $g$ is called a $p$-weak upper gradient for $f$. When the exponent $p$ is clear, we omit it.

The concept of upper gradient was introduced in 12 . It was initially called "very weak gradient", but the befitting term "upper gradient" was soon suggested. Functions with $p$-integrable $p$-weak upper gradients were subsequently studied in [26], while the theory of Sobolev spaces based on upper gradient was systematically developed in 37] and [4.

By [15, Lemma 5.2.3], $f$ has a $p$-weak upper gradient in $L_{l o c}^{p}(X)$ if and only if it has an actual upper gradient in $L_{l o c}^{p}(X)$.

A $p$-weak upper gradient $g$ of $f$ is minimal if for every $p$-weak upper gradient $\tilde{g}$ of $f, \tilde{g} \geq g \mu$-almost everywhere. If $f$ has an upper gradient in $L_{\text {loc }}^{p}(X)$, then $f$ has a unique (up to sets of $\mu$-measure zero) minimal $p$-weak upper gradient by the following result from [15, Theorem 5.3.23]. In this situation, we denote the minimal upper gradient by $g_{f}$.

Proposition 2.4. The collection of all p-integrable $p$-weak upper gradients of a map $u: X \rightarrow Z$ is a closed convex lattice inside $L^{p}(X)$ and, if nonempty, contains a unique element of smallest $L^{p}$-norm. In particular, if a map has a p-integrable p-weak upper gradient, then it has a minimal p-weak upper gradient. 
In view of the above result, the minimal $p$-weak upper gradient $\rho_{u}$ should be thought of as a substitute for $|\nabla u|$, or the length of a gradient, for functions defined in metric measure spaces.

Let $\tilde{N}^{1, p}(X, Z)$ denote the collection of all maps $u \in L^{p}(X, Z)$ that have an upper gradient in $L^{p}(X)$. We equip it with seminorm

$$
\|u\|_{\tilde{N}^{1, p}(X, Z)}=\|u\|_{L^{p}(X, Z)}+\left\|g_{u}\right\|_{L^{p}(X)},
$$

where $g_{u}$ is the minimal $p$-weak upper gradient of $u$ guaranteed by Proposition 2.4.

We obtain a normed space $N^{1, p}(X, Z)$ by passing to equivalence classes of functions in $\tilde{N}^{1, p}(X, Z)$, where $u_{1} \sim u_{2}$ if and only if $\left\|u_{1}-u_{2}\right\|_{\tilde{N}^{1, p}(X, Z)}=0$. Thus

$$
N^{1, p}(X, Z):=\tilde{N}^{1, p}(X, Z) /\left\{u \in \tilde{N}^{1, p}(X, Z):\|u\|_{\tilde{N}^{1, p}(X, Z)}=0\right\} .
$$

Let $\tilde{N}_{l o c}^{1, p}(X, Z)$ be the vector space of functions $u: X \rightarrow Z$ with the property that every point $x \in X$ has a neighborhood $U_{x}$ in $X$ such that $u \in \tilde{N}^{1, p}\left(U_{x}, Z\right)$. Two functions $u_{1}$ and $u_{2}$ in $\tilde{N}_{l o c}^{1, p}(X, Z)$ are said to be equivalent if every point $x \in X$ has a neighborhood $U_{x}$ in $X$ such that the restrictions $\left.u_{1}\right|_{U_{x}}$ and $\left.u_{2}\right|_{U_{x}}$ determine the same element in $\tilde{N}^{1, p}\left(U_{x}, Z\right)$. The local Sobolev space $N_{l o c}^{1, p}(X, Z)$ is the vector space of equivalent classes of functions in $\tilde{N}_{l o c}^{1, p}(X, Z)$ under the preceding equivalence relation.

With the notion of upper gradient, we are now ready to define the p-capacity of a general condenser in a metric measure space $(X, d, \mu)$.

Suppose $E$ and $F$ are closed subsets of an open set $\Omega$ in $X$. The triple $(E, F, \Omega)$ is called a condenser and its $p$-capacity for $1 \leq p<\infty$ is defined as

$$
\operatorname{Cap}_{p}(E, F, \Omega)=\inf \int_{\Omega} g^{p} d \mu,
$$

where the infimum is taken over all $p$-weak upper gradients of all functions $u$ in $\Omega$ such that $\left.u\right|_{E} \geq 1$ and $\left.u\right|_{F} \leq 0$. Such a function $u$ is called admissible for the condenser $(E, F, U)$.

Note that in the definition of $p$-capacity, we do not require any regularity of admissible functions. However, the existence of $L^{p}$-weak upper gradients in practice imposes restrictions. In particular, for a proper doubling metric measure space supporting a Poincaré inequality (in the sense of the next section), one can use locally Lipschitz continuous function as admissible functions for capacity; see 13 , Proposition 2.4] or [12, Proposition 2.17]. Moreover, the following equation holds:

$$
\operatorname{Cap}_{p}(E, F, \Omega)=\operatorname{Mod}_{p}(E, F, \Omega) .
$$

\subsection{Poincaré inequalities and consequences.}

Definition 2.5. We say that a metric measure space $(X, d, \mu)$ admits a $(1, p)$ Poincaré inequality if there exist constants $C \geq 1$ and $\tau \geq 1$ such that

$$
f_{B}\left|u-u_{B}\right| d \mu \leq C \operatorname{diam}(B)\left(f_{\tau B} g^{p} d \mu\right)^{1 / p}
$$

for all open balls $B$ in $X$, for every function $u: X \rightarrow \mathbb{R}$ that is integrable on balls and for every upper gradient $g$ of $u$ in $X$.

For a complete doubling metric measure space $X=(X, d, \mu)$ supporting a $(1, p)$ Poincaré inequality, there are many important consequences. For example, one has 
the important Sobolev embedding as in the Euclidean case. We need the following fact from [15, Theorem 7.3.2]; see also [8].

Lemma 2.6. A complete and doubling metric measure space that supports a Poincaré inequality is quasiconvex.

Recall that a metric space $Z=\left(Z, d_{Z}\right)$ is said to be $C$-quasiconvex or simply quasiconvex, $C \geq 1$, if each pair of points can be joined by a rectifiable curve in $Z$ such that

$$
l(\gamma) \leq C d_{Z}(x, y)
$$

Note that for a doubling metric space, completeness is equivalent to properness (cf. 2, Proposition 3.1]).

Recall that a metric space $X$ is said to be $\theta$-linearly locally connected ( $\theta$-LLC) if there exists $\theta \geq 1$ such that for $x \in X$ and $0<r \leq \operatorname{diam}(X)$ :

(i) every two points $a, b \in B(x, r)$ can be joined in $B(x, \theta r)$, and

(ii) every two points $a, b \in X \backslash \bar{B}(x, r)$ can be joined in $X \backslash \bar{B}\left(x, \theta^{-1} r\right)$.

Here, by joining $a$ and $b$ in $B$ we mean that there exists a path $\gamma:[0,1] \rightarrow B$ with $\gamma(0)=a, \gamma(1)=b$.

Remark 2.7. Note that in the above definition, instead of a continuum, we choose to use a curve to join two points. This is in a prior stronger assumption. However, by [19, Theorem 3-17] a connected, locally connected, complete metric space is arcwise connected. Hence for such spaces, joining by continuum and joining by curve (or arc) are equivalent.

Based on Remark 2.7 throughout the paper, we assume that all the metric spaces in question are connected and locally connected.

The following result was proved in [12, Corollary 5.8], where the quasiconvexity condition is provided by Lemma 2.6

Proposition 2.8. Let $(X, d, \mu)$ be a complete Ahlfors n-regular metric measure space that supports a $(1, n)$-Poincaré inequality. Then $X$ is $\theta$-linearly locally connected with $\theta$ depending only on the data associated with $X$.

Definition 2.9. Let $(X, d, \mu)$ be a metric measure space. We say that $X$ has $n$-Loewner property if there is a function $\phi:(0, \infty) \rightarrow(0, \infty)$ such that

$$
\operatorname{Mod}_{n}(\Gamma(E, F, X)) \geq \phi(\zeta(E, F))
$$

for every nondegenerate compact connected sets $E, F \subset X$, where

$$
\zeta(E, F)=\frac{\operatorname{dist}(E, F)}{\min \{\operatorname{diam} E, \operatorname{diam} F\}} .
$$

By [12, Theorem 3.6], if $X$ is Ahlfors $n$-regular, and $n$-Loewner, then

$$
\operatorname{Mod}_{n}(\Gamma(E, F, X)) \geq C(\log \zeta(E, F))^{1-n}
$$

when $\zeta(E, F)$ is large enough with $C$ depends only on the data of $X$. By [12, Corollary 5.13], a complete (or equivalently proper) Ahlfors $n$-regular metric measure space that supports a $(1, n)$-Poincaré inequality is $n$-Loewner. 


\section{MAPPINGS OF Finite Distortion}

In this section, we define mappings of finite distortion between certain metric measure spaces. The metric measure spaces $\left(X, d_{X}, \mu\right)$ and $\left(Y, d_{Y}, \nu\right)$ in this paper, unless specialized, are always assumed to be proper, Ahlfors $n$-regular, and support a $(1,1)$-Poincaré inequality. We will always use the symbol $\Omega$ to denote a domain in $X$ and in most situations drop the subscripts in the metrics $d_{X}$ and $d_{Y}$ when the context is clear. Without loss of generality, we only consider the case $\mu=\nu=\mathscr{H}^{n}$ in most situations.

Recall that a mapping $f: X \rightarrow Y$ between topological spaces is discrete if each fiber is a discrete set in $X$, i.e. for all $y \in Y, f^{-1}(y)$ is a discrete set in $X$, and is open if it maps an open set in $X$ onto an open set in $Y$. For a mapping $f: X \rightarrow Y$ and a subset $A \subset X$, we will use the notation

$$
N(y, f, A)=\#\left\{f^{-1}(y) \cap A\right\}
$$

for the multiplicity function of $f$. We also write $N(f, A):=\sup _{y \in Y} N(y, f, A)$. A mapping $f: X \rightarrow Y$ is said to have locally bounded multiplicity if every point $x \in X$ has a neighborhood $U \subset X$ such that $N(f, U)<\infty$, and has bounded multiplicity if $N(f, X)<\infty$.

Let $f: X \rightarrow Y$ be a continuous, discrete and open mapping of locally bounded multiplicity between two locally compact metric measure spaces. We define a locally finite Borel regular outer measure (so-called pull-back measure) as

$$
f^{*} \mathscr{H}^{n}(A)=\int_{Y} N(y, f, A) d \mathscr{H}^{n}(y) .
$$

The metric "Jacobian" above is given by

$$
J_{f}(x)=\frac{d\left(f^{*} \mathscr{H}^{n}\right)(x)}{d \mathscr{H}^{n}(x)} .
$$

Definition 3.1. A continuous mapping $f: \Omega \rightarrow Y$ is called a mapping of finite distortion if $f$ is either constant or

- $f$ is discrete, open, and of locally bounded multiplicity;

- $f \in N_{l o c}^{1, n}(\Omega, Y)$;

- there is a measurable function $K=K(x) \geq 1$, finite almost everywhere, such that $f$ satisfies the distortion inequality

$$
g_{f}(x)^{n} \leq K(x) J_{f}(x) \text { a.e. } \Omega .
$$

If $K(\cdot) \in L^{\infty}(\Omega)$, then we call $f K$-quasiregular or simply quasiregular.

Next we introduce an useful concept called pseudomonotonicity.

Definition 3.2. A mapping $f: \Omega \rightarrow Y$ is said to be $T$-pseudomonotone or simply pseudomonotone if there exists a constant $T \geq 1$ such that

$$
\operatorname{diam} f(B(x, r)) \leq T \operatorname{diam} f(S(x, r))
$$

for every $B(x, r) \subset \subset \Omega$. If $T$ happens to be 1 , then $f$ is called monotone.

Lemma 3.3. Suppose that $f: \Omega \rightarrow Y$ is continuous and open. Then for every $x \in \Omega$, there exists a radius $R=R(x)>0$ such that $\left.f\right|_{B(x, R)}$ is T-pseudomonotone, where $T \geq 1$ only depends on the data. 
Proof. By Proposition 2.8, we may assume that $X$ and $Y$ are $C$-linearly locally connected. Fix $x \in \Omega$. Since $f$ is open, it is nonconstant. Thus, by the continuity of $f$, there exists $0<R<1$ and $p \in \Omega$ such that $B\left(x, C^{2} R\right) \subset \subset \Omega$, $5 \operatorname{diam} f\left(B\left(x, C^{2} R\right)\right)<\frac{\operatorname{diam}(Y)}{C}$ and

$$
f(p) \in Y \backslash B(f(x), 5 \operatorname{diam} f(B(x, R))) .
$$

Now fix $B=B(y, r) \subset B(x, R)$ and a point $\omega \in \partial f(B)$. Recall that the openness of $f$ implies that $\partial f(B) \subset f(\partial B)$, and thus

$$
\operatorname{diam} f(\partial B) \geq \operatorname{diam} \partial f(B)=: A .
$$

Then

$$
\operatorname{diam} f(B)=\sup _{u, v \in f(B)} d(u, v) \leq \sup _{u, v \in f(B)}(d(u, \omega)+d(v, \omega))=2 \sup _{v \in f(B)} d(v, \omega) .
$$

To complete the proof, we show that, given $v \in f(B), d(v, \omega) \leq C A$, where $C \geq 1$ does not depend on $y$ or $r$.

Fix a constant $1 \leq M \leq 2 \operatorname{diam} f(B) / A$ such that

$$
v \notin B(\omega, M A) .
$$

Since $\omega \in \partial f(B) \subset f(\partial B)$, there exists a point $\omega^{\prime} \in \partial B$ such that $f\left(\omega^{\prime}\right)=\omega$. Thus, by (3.6) and the LLC-property of $X$, there exists a path

$$
\gamma:[0,1] \rightarrow\left(\Omega \backslash C^{-1} \bar{B}\right) \cup\left\{\omega^{\prime}\right\}
$$

such that $\gamma(0)=p$ and $\gamma(1)=\omega^{\prime}$. We conclude that there exists a point $u \in f(|\gamma|)$ such that

$$
u \in B(\omega, 3 \operatorname{diam} f(B)) \backslash(B(\omega, M A) \cup f(\bar{B})) .
$$

By (3.7), (3.8), and the LLC-property of $Y$, there exists a path

$$
\alpha:[0,1] \rightarrow Y \backslash B\left(\omega, C^{-1} M A\right)
$$

such that $\alpha(0)=v \in f(B)$ and $\alpha(1)=u \notin \overline{f(B)}$. But then $M \leq C$, since otherwise $|\alpha| \cap \partial f(B)=\emptyset$, which contradicts the connectedness of $\alpha$. We conclude that $v \in B(\omega, M A)$ for every $M \geq C$, i.e. $d(v, \omega) \leq 2 C$. The proof is complete.

We end this section by pointing out the following modulus estimate, which is needed in the proof of [16, Lemma 6.19].

Lemma 3.4. Let $X$ be a proper, Ahlfors $n$-regular metric measure space supporting $a(1, n)$-Poincaré inequality. Suppose that $0<r<R$ and that $E, F \subset B(x, R) \subset X$ are disjoint compact sets such that

$$
E \cap S(x, s) \neq \emptyset, F \cap S(x, s) \neq \emptyset
$$

for every $s \in(r, R)$. Then there is a constant $C_{0}$ that depends on the data of $X$ such that $R \geq C_{0} r$ implies

$$
\operatorname{Mod}_{n}(\Gamma) \geq C_{0}^{-1} \log \frac{R}{r},
$$

where $\Gamma$ is the family of curves in $B(x, R)$ joining $E$ and $F$. 
Proof. As pointed out earlier, by [13, Proposition 2.4] or [12, Proposition 2.17], one can use locally Lipschitz functions (in particular $N^{1, n}(B(x, R))$-function) as admissible functions for the capacity. Let $u \in N^{1, n}(B(x, R))$ be an admissible function for $\Gamma$ and $g_{u}$ the minimal $n$-weak upper gradient of $u$. Then $\left(u, g_{u}\right)$ satisfies the $(1, \mathrm{n})$-Poincaré inequality. Moreover, by Proposition 2.8 and Lemma 2.6. we know that $B(x, R)$ is $C$-LLC. Hence [8, Corollary 7.3] implies that

$$
|u(x)-u(y)| \leq C r_{0}\left(f_{C B\left(x, r_{0}\right) \backslash C^{-1} B\left(x, r_{0}\right)} g_{u}^{n} d \mathscr{H}^{n}\right)^{1 / n}
$$

for all $x, y \in S\left(x, r_{0}\right)$ and $r \leq r_{0} \leq R$. Thus, if we choose $x \in E$ and $y \in F$ on $S\left(x, r_{0}\right)$ in (3.10), then we obtain

$$
\int_{C B\left(x, r_{0}\right) \backslash C^{-1} B\left(x, r_{0}\right)} g_{u}^{n} d \mathscr{H}^{n} \geq C_{0} .
$$

Let $C_{0} \geq C^{2}$. Then let $A_{i}$ be the "annulus" defined by

$$
A_{i}=\bar{B}\left(x, 2^{i} C r\right) \backslash B\left(x, 2^{i-1} C^{-1} r\right), i=2, \ldots,\left[\log \frac{R}{C r}\right] .
$$

Let $\Gamma_{i}$ be the family of curves in $A_{i}$ joining $E$ and $F$. Then we obtain

$$
\operatorname{Mod}_{n}(\Gamma) \geq \sum_{i=2}^{\left[\log \frac{R}{C r}\right]} \operatorname{Mod}_{n}\left(\Gamma_{i}\right) \geq C_{0}\left(\log \frac{R}{C r}-2\right) \geq C^{\prime} \log \frac{R}{r}
$$

as desired.

\section{Analytic properties of mappings of Finite Distortion}

4.1. Condition $\mathbf{N}$ and area formula. In this subsection, we show that a mapping $f: \Omega \rightarrow Y$ of finite distortion satisfies Condition $N$, i.e. $f$ maps sets of zero $n$ Hausdorff measure to sets of zero $n$-Hausdorff measure, and that the change of variable formula holds for $f$.

We begin with the following proposition, which shows the usefulness of pseudomonotonicity. It was proved in greater generality in [14, Theorem 7.2].

Proposition 4.1. If $f: \Omega \rightarrow Y$ is a pseudomonotone map in $N_{l o c}^{1, n}(\Omega, Y)$, then $f$ satisfies Condition $N$.

Since Condition $\mathrm{N}$ is a local property, we obtain the following corollary.

Corollary 4.2. If $f: \Omega \rightarrow Y$ is mapping of finite distortion, then $f$ satisfies Condition $N$.

In order to prove (4.1) for mappings of finite distortion, we need the following properties of mappings in Newtonian spaces from [14, Proposition 4.6].

Lemma 4.3. If $f: \Omega \rightarrow Y$ belongs to $N_{\text {loc }}^{1, p}(\Omega, Y)$ for some $1 \leq p<\infty$, then there exists a partition

$$
\Omega=\left(\bigcup_{k=1}^{\infty} A_{k}\right) \cup E, \quad E, A_{1}, A_{2}, \ldots \text { pairwise disjoint }
$$

such that $\left.f\right|_{A_{k}}$ is $k$-Lipschitz for every $k \in \mathbb{N}$ and $\mathscr{H}^{n}(E)=0$. In particular, if $f$ satisfies Condition $N$ and $\Omega$ is n-rectifiable with $n \in \mathbb{N}$, then $f(\Omega)$ is n-rectifiable. 
Recall that a set $E \subset X$ is $n$-rectifiable if $E$ has a countable union of Lipschitz images of the subset of $\mathbb{R}^{n}$ plus a set of Hausdorff $n$-measure zero.

The following well-known change of variable formula (area formula) for Lipschitz mappings was proved in [28, Theorem 1].

Proposition 4.4. Let $f: X \rightarrow Y$ be a Lipschitz mapping. Then the area formula

$$
\int_{X} u \circ f(x) J_{f}(x) d \mathscr{H}^{n}(x)=\int_{Y} u(y) N(y, f, X) d \mathscr{H}^{n}(y)
$$

holds for each nonnegative measurable function $u$ on $Y$.

Corollary 4.5. Suppose that $f: \Omega \rightarrow Y$ is map in $N_{l o c}^{1, p}(\Omega, Y)$ satisfying Condition $N$. Then (4.1) is valid. Moreover, if $\Omega$ is n-rectifiable with $n \in \mathbb{N}$, then $f(\Omega)$ is also $n$-rectifiable. In particular, the results hold for mappings of finite distortion.

Proof. Note that (4.1) holds true for the restrictions $\left.f\right|_{A_{k}}$ given in Lemma 4.3. Thus $\mathscr{H}^{n}(E)=\mathscr{H}^{n}(f(E))=0$, by Corollary 4.2 and Lemma 4.3. Therefore, (4.1) follows by applying the formula for each $\left.f\right|_{A_{k}}$ and summing both sides over $k$. The second claim is clear from Corollary 4.2 and Lemma 4.3

We end this subsection with the following simple fact.

Proposition 4.6. Suppose that $f: \Omega \rightarrow Y$ is a mapping of finite distortion. Then

$$
\operatorname{Lip} f(x)<\infty
$$

for almost every $x \in \Omega$, where $\operatorname{Lip} f$ is the pointwise (upper) Lipschitz constant function of $f$ given by

$$
\operatorname{Lip} f(x):=\limsup _{r \rightarrow 0} \sup _{y \in B(x, r)} \frac{d(f(x), f(y))}{r} .
$$

Proof. Note that the issue is local and the conclusion is bi-Lipschitz invariant, we may assume that $Y$ is a subset of a Banach space. Then Lemma 3.3 and the proof of [14, Theorem 7.2] imply that there is a constant $\delta>0$ such that for balls $B(x, r) \subset \Omega$ with $0<r<\delta$ we have

$$
\operatorname{diam} f(B(x, r))^{n} \leq C \int_{B(x, r)} g_{f}^{n}(y) d \mathscr{H}^{n}(y),
$$

where $C$ is a constant depending only on the data of $\Omega$ and $Y$. The claim follows immediately from the Lebesgue differentiation theorem.

4.2. Differentiability of mappings of finite distortion. In this subsection, we consider differentiability result for mappings of finite distortion. We recall first the definition of strong measurable differentiable structure of a metric measure space.

Definition 4.7. Let $(X, d, \mu)$ be a metric measure space, let $\mathscr{C} \subset \operatorname{LIP}(X)$ be a vector space of functions, and let $\left\{\left(X_{\alpha}, \varphi_{\alpha}\right)\right\}$ be a countable collection of pairs $\left(X_{\alpha}, \varphi_{\alpha}\right)$, called coordinate patches, such that each $X_{\alpha} \subset X$ is measurable with positive measure, and such that each

$$
\varphi_{\alpha}=\left(\varphi_{\alpha}^{1}, \ldots, \varphi_{\alpha}^{N}\right): X \rightarrow \mathbb{R}^{N(\alpha)}
$$

is a function for some $N(\alpha) \in \mathbb{N} \cup\{0\}$, where $\varphi_{\alpha}^{i} \in \mathscr{C}$ for every $1 \leq i \leq N(\alpha)$. Then $\left\{\left(X_{\alpha}, \varphi_{\alpha}\right)\right\}$ is said to be a strong measurable differentiable structure for $(X, d, \mu)$ with respect to $\mathscr{C}$ if the following conditions are true. 
i) The sets $X_{\alpha}$ are pairwise disjoint and

$$
\mu\left(X \backslash \bigcup_{\alpha} X_{\alpha}\right)=0
$$

ii) There exists a number $N \geq 0$ such that $N(\alpha) \leq N$ for every coordinate patch $\left(X_{\alpha}, \varphi_{\alpha}\right)$.

iii) For every $f \in \mathscr{C}$ and coordinate patch $\left(X_{\alpha}, \varphi_{\alpha}\right)$, there is a unique (up to a set of measure zero) measurable function $d f^{\alpha}: X_{\alpha} \rightarrow \mathbb{R}^{N(\alpha)}$ such that for $\mu$-almost every $x \in X_{\alpha}$,

$$
\lim _{y \rightarrow x, y \neq x} \frac{\left|f(y)-f(x)-d f^{\alpha}(x) \cdot\left(\varphi_{\alpha}(y)-\varphi_{\alpha}(x)\right)\right|}{d(y, x)}=0 .
$$

The follow well-known result is proved by Cheeger [4].

Proposition 4.8. Let $(X, d, \mu)$ be a complete doubling metric measure space supporting a $(1, p)$-Poincaré inequality for some $p \geq 1$. Then $(X, d, \mu)$ admits a strong measurable differentiable structure for $\operatorname{Lip}(X)$.

We are now able to define the notion of Cheeger's differentiability.

Definition 4.9. Let $\left\{\left(X_{\alpha}, \varphi_{\alpha}\right)\right\}$ be a strong measurable differentiability structure on a metric measure space $(X, d, \mu)$ and $V$ be a Banach space. Given a measurable subset $S$ of $X$, a measurable mapping $f: S \rightarrow V$ is approximately differentiable at $x \in X_{\alpha} \cap S$ with respect to $\left(X_{\alpha}, \varphi_{\alpha}\right)$ if there exists a unique (up to set of measure zero) collection of measurable mappings $\left\{\frac{\partial f}{\partial \varphi_{n}^{\alpha}}: X_{\alpha} \cap S \rightarrow V\right\}$ such that

$$
\text { ap } \lim _{y \rightarrow x} \frac{\left|f(y)-f(x)-\sum_{n=1}^{N(\alpha)}\left(\varphi_{n}^{\alpha}(y)-\varphi_{n}^{\alpha}(x)\right) \frac{\partial f}{\partial \varphi_{n}^{\alpha}}(x)\right|}{d(y, x)}=0,
$$

i.e. for every $\varepsilon>0$ the set

$$
A_{x, \varepsilon}=\left\{y \in X: \frac{\left|f(y)-f(x)-\sum_{n=1}^{N(\alpha)}\left(\varphi_{n}^{\alpha}(y)-\varphi_{n}^{\alpha}(x)\right) \frac{\partial f}{\partial \varphi_{n}^{\alpha}}(x)\right|}{d(y, x)}<\varepsilon\right\}
$$

has $x$ as a density point. If we change the approximate limit in (4.5) to limit, then $f$ is said to be differentiable at $x$ with respect to $\left(X_{\alpha}, \varphi_{\alpha}\right)$. Note that in the formulation of (4.5), we have used the symbol $|\cdot|$ to represent the Banach norm of $V$.

One can easily prove that the approximate differential or differential is welldefined, in the sense that if there exists a collection of measurable mappings $\left\{\frac{\partial f}{\partial \varphi_{n}^{\alpha}}\right.$ : $\left.X_{\alpha} \rightarrow V\right\}$ satisfying (4.5) then it is unique for almost all points $x \in X_{\alpha}$; see [6. Lemma 1.10].

Definition 4.10. A Banach space $V$ is said to have the Radon-Nikodym property if every Lipschitz map $f: \mathbb{R} \rightarrow V$ is differentiable Lebesgue almost everywhere.

Cheeger and Kleiner [5] proved that if $X$ is a doubling metric measure space that supports a $p$-Poincaré inequality for some $1 \leq p<\infty$, and $\left\{\left(X_{\alpha}, \varphi_{\alpha}\right)\right\}$ is a strong measurable differentiable structure on $X$, then every Lipschitz mapping from $X$ to a Banach space with the Radon-Nikodym property is differentiable almost everywhere in $X$ with respect to the strong measurable structure. 
We next show that the notion of approximately differentiability or differentiability does not depend on the choice of the strong measurable differentiable structure if the target $V$ has the Radon-Nikodym property. The proof is similar to that used in [6. Lemma 1.13].

Lemma 4.11. Let $(X, d, \mu)$ be a metric measure space that admits a strong measurable differentiable structure $\left\{\left(X_{\alpha}, \varphi_{\alpha}\right)\right\}$ and $V$ has the Radon-Nikodym property. If $f: X \rightarrow V$ is approximately differentiable (or differentiable) $\mu$-a.e. with respect to $\left\{\left(X_{\alpha}, \varphi_{\alpha}\right)\right\}$, then it is approximately differentiable (or differentiable) $\mu$-a.e. with respect to any strong differentiable structure defined on $X$.

Proof. Let $\left\{\left(X_{\alpha}, \varphi_{\alpha}\right)\right\}$ and $\left\{\left(X_{\beta}, \varphi_{\beta}\right)\right\}$ be two strong measurable differentiable structures defined on $(X, d, \mu)$. We write $\left\{\frac{\partial f}{\partial \varphi_{n}^{\alpha}}\right\}$ for the approximate differential of $f$ with respect to $\left\{\left(X_{\alpha}, \varphi_{\alpha}\right)\right\}$ at $x \in X_{\alpha}$.

For fixed $x$, the Banach-valued map $g_{x}(\cdot)=\sum_{n=1}^{N(\alpha)} \frac{\partial f}{\partial \varphi_{n}^{\alpha}}(x) \varphi_{n}^{\alpha}(\cdot)$ is a Lipschitz map from $X$ to $V$, thus it is approximately differentiable $\mu$-a.e. with respect to $\left\{\left(X_{\beta}, \varphi_{\beta}\right)\right\}$. Moreover, the set of points where $g_{x}$ is approximately differentiable does not depend on the choice of $x$.

For almost every $x \in X_{\beta}$ we can choose $\alpha$ such that $x \in X_{\alpha}$ and $x$ is a point of approximately differentiability of $f$ with respect to $\left\{\left(X_{\alpha}, \varphi_{\alpha}\right)\right\}$. Thus for $\mu$-a.e. $x \in X_{\beta}$ we have

$$
\begin{aligned}
& \left|f(y)-f(x)-\sum_{n=1}^{N(\beta)}\left(\varphi_{n}^{\beta}(y)-\varphi_{n}^{\beta}(x)\right) \frac{\partial g_{x}}{\partial \varphi_{n}^{\beta}}(x)\right| \\
& \leq\left|f(y)-f(x)-\sum_{n=1}^{N(\alpha)}\left(\varphi_{n}^{\alpha}(y)-\varphi_{n}^{\alpha}(x)\right) \frac{\partial f}{\partial \varphi_{n}^{\alpha}}(x)\right| \\
& \quad+\left|g_{x}(y)-g_{x}(x)-\sum_{n=1}^{N(\beta)}\left(\varphi_{n}^{\beta}(y)-\varphi_{n}^{\beta}(x)\right) \frac{\partial g_{x}}{\partial \varphi_{n}^{\beta}}(x)\right| .
\end{aligned}
$$

It follows easily from the above inequality that $x$ is a point of approximately differentiability of $f$ with respect to $\left\{\left(X_{\beta}, \varphi_{\beta}\right)\right\}$. Hence we conclude that $f$ is approximately differentiable $\mu$-a.e. on $X$ with respect to $\left\{\left(X_{\beta}, \varphi_{\beta}\right)\right\}$.

Next we generalize the main results of [6] to Banach-valued mappings.

Theorem 4.12. Let $(X, d, \mu)$ be a complete doubling metric measure space that supports a $(1, p)$-Poincaré inequality for some $1 \leq p<\infty$, and $V$ be a Banach space with Radon-Nikodym property. Suppose that $E \subset X$ is measurable and $f: E \rightarrow V$ is measurable. Then the following conditions are equivalent:

(1) $f$ is approximately differentiable $\mu$-a.e. in $E$ with respect to the strong measurable differentiable structure.

(2) For any $\varepsilon>0$ there is a closed set $F \subset E$ such that $\mu(E \backslash F)<\varepsilon$ and $\left.f\right|_{F}$ is locally Lipschitz.

(3) $f$ induces a Lusin decomposition of $E$, that is,

$$
E=\bigcup_{j=1}^{\infty} E_{j} \cup Z,
$$

where $E_{j}$ are measurable sets, $\left.f\right|_{E_{i}}$ are Lipschitz mappings and $Z$ has measure zero. 
Proof. The proof is similar to that used in [6, Theorem 2.1]. Let $\left\{\left(X_{\alpha}, \varphi_{\alpha}\right)\right\}$ be a strong measurable differentiable structure defined on $(X, d, \mu)$. Let $f$ be approximately differentiable $\mu$-a.e. in $E$. Let $|D f|_{\alpha}(x)=\sum_{n=1}^{N(\alpha)}\left|\frac{\partial f}{\partial \varphi_{n}^{\alpha}}(x)\right|$. Then $|D f|_{\alpha}(\cdot)$ is a measurable function defined on $X$ (in prior $|D f|_{\alpha}(\cdot)$ is only defined on $X_{\alpha}$, but one can extend it to be zero in $\left.X \backslash X_{\alpha}\right)$. Finally, we let $|D f|(x)=\sum_{\alpha}|D f|_{\alpha}(x)$. Clearly, $|D f|$ is also a measurable function defined on $X$.

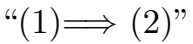

First, assume that $E \subset X$ is bounded and define

$$
D=\{x \in E: f \text { is approximately differentiable at } x\} .
$$

We want to show that for any $\varepsilon>0$ there exists a closed set $F=F_{\varepsilon} \subset D, \delta>0$ and $L>0$ such that $\mu(D \backslash F)<\varepsilon$ and

$$
|f(x)-f(y)| \leq L d(x, y)
$$

for each $x, y \in F$ such that $d(x, y)<\delta$.

Since $\mu$ is a doubling measure, for any $r>0, x, y \in X$ such that $d(x, y) \leq r / 2$,

$$
\mu(B(x, r) \cap B(y, r)) \geq \mu(B(x, r / 2)) \geq 2 a \mu(B(x, r)),
$$

where $a=\frac{1}{2 C_{\mu}}$ and $C_{\mu}$ is the doubling constant.

For any $\eta>0$ define the following sequence of functions:

$$
\psi_{i}^{n}(x)=\mu\left(B(x, 1 / i) \backslash A_{x, \eta}\right) \quad x \in D, \quad i \in \mathbb{N},
$$

where $A_{x, \eta}$ is given by formula (4.6). It is easy to see that for each $\eta>0$ and $x \in D$ one has

$$
\phi_{i}^{n}(x)=\frac{\psi_{i}^{n}(x)}{\mu(B(x, 1 / i))} \rightarrow 0 \text { as } i \rightarrow \infty .
$$

Next, set $\eta=1$. By Luzin's and Egoroff's theorem there exists a closed set $F \subset E$ such that

i: $\mu(E \backslash F)=\mu(D \backslash F)<\varepsilon$,

ii: $\mid D f \|_{F}$ is continuous, moreover, since $X$ is proper, $\mid D f \|_{F}$ is bounded in $F$, i.e. $\mid D f \|_{F} \leq C$,

iii: $\phi_{i}^{1} \rightarrow 0$ uniformly on $F$.

Choose $i_{0}$ such that

$$
\phi_{i}^{1}(x)<\frac{a}{C_{\mu}}, x \in F, i \geq i_{0} .
$$

Fix $x, y \in F$ such that $d(x, y)<\frac{1}{2 i_{0}}$. Points $x$ and $y$ may belong to two different charts, we write $x \in X_{\alpha}$ and $y \in X_{\beta}$. Choose $i \geq i_{0}$ such that

$$
\frac{1}{2(i+1)}<d(x, y) \leq \frac{1}{2 i} \text {. }
$$

For such $i$ we have that $i \geq i_{0}$ and

$$
\psi_{i}^{1}(y)<\frac{a}{C_{\mu}} \mu(B(y, 1 / i)) \leq \frac{a}{C_{\mu}} \mu(B(x, 2 / i)) \leq a \mu(B(x, 1 / i)) .
$$

Hence

$$
\psi_{i}^{1}(x)<a \mu(B(x, 1 / i)) \text { and } \psi_{i}^{1}(y)<a \mu(B(x, 1 / i)) .
$$


Combining (4.8) and (4.9), we deduce that there exists a point $z \in B(x, 1 / i) \cap$ $B(y, 1 / i)$ which does not belong to either $B(x, 1 / i) \backslash A_{x, 1}$ or $B(y, 1 / i) \backslash A_{y, 1}$. For such a point $z$, we have $d(x, z)<1 / i, d(y, z)<1 / i$,

$$
\frac{\left|f(z)-f(x)-\sum_{n=1}^{N(\alpha)}\left(\varphi_{n}^{\alpha}(z)-\varphi_{n}^{\alpha}(x)\right) \frac{\partial f}{\partial \varphi_{n}^{\alpha}}(x)\right|}{d(z, x)}<1
$$

and

$$
\frac{\left|f(z)-f(y)-\sum_{n=1}^{N(\beta)}\left(\varphi_{n}^{\beta}(z)-\varphi_{n}^{\beta}(y)\right) \frac{\partial f}{\partial \varphi_{n}^{\beta}}(y)\right|}{d(z, y)}<1 .
$$

By combining (4.10), 4.11), $d(y, z)<5 d(x, y)$ and $d(x, z)<5 d(x, y)$ we obtain

$$
\begin{aligned}
|f(y)-f(x)| \leq \mid & f(z)-f(x)-\sum_{n=1}^{N(\alpha)}\left(\varphi_{n}^{\alpha}(z)-\varphi_{n}^{\alpha}(x)\right) \frac{\partial f}{\partial \varphi_{n}^{\alpha}}(x) \mid \\
& \quad+\left|f(z)-f(y)-\sum_{n=1}^{N(\beta)}\left(\varphi_{n}^{\beta}(z)-\varphi_{n}^{\beta}(y)\right) \frac{\partial f}{\partial \varphi_{n}^{\beta}}(y)\right| \\
& +\left|\sum_{n=1}^{N(\alpha)}\left(\varphi_{n}^{\alpha}(z)-\varphi_{n}^{\alpha}(x)\right) \frac{\partial f}{\partial \varphi_{n}^{\alpha}}(x)\right|+\left|\sum_{n=1}^{N(\beta)}\left(\varphi_{n}^{\beta}(z)-\varphi_{n}^{\beta}(y)\right) \frac{\partial f}{\partial \varphi_{n}^{\beta}}(y)\right| \\
& \leq 10(1+C) d(x, y),
\end{aligned}
$$

which shows that $\left.f\right|_{F}$ is locally Lipschitz and finishes the proof of the implication for the case $E \subset X$ bounded.

The corresponding arguments in [6] allow us to proceed to the general case and thus the claim holds.

$$
\text { "(2) } \Longrightarrow(3) "
$$

The same proof as in [6].

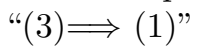

If decomposition (4.7) holds, then the restriction $\left.f\right|_{E_{i}}$ is Lipschitz for every $i$. By [15. Theorem 3.1.25], we can extend $\left.f\right|_{E_{i}}$ to a Lipschitz map $\tilde{f}_{i}$ defined on the whole space $X$. Then by the results in [5], $\tilde{f}_{i}$ is (approximately) differentiable $\mu$-a.e. with respect to the strong measurable differentiable structure on $X$. Since every point of a measurable set $E_{i}$ is its point of density, $f$ is also approximately differentiable $\mu$-a.e. on $E_{i}$ with respect to the strong measurable differentiable structure on $X$.

Remark 4.13. It is easily seen from the proof that if $E \subset X$ is bounded, then condition (2) in Theorem 4.12 can be replaced by:

(2') For any $\varepsilon>0$ there exists a closed set $F \subset E$ and a Lipschitz map $g: X \rightarrow V$ such that $\mu(E \backslash F)<\varepsilon$ and $\left.f\right|_{F}=g$.

The following Stepanov-type theorem is essentially proved in [6, Theorem 2.3] (it is there stated for $\mathbb{R}$-valued functions, but the proof works for $V$-valued mappings as well).

Theorem 4.14. Let $(X, d, \mu)$ be a complete doubling metric measure space and $V$ be a Banach space. A measurable map $f: E \rightarrow V$ defined on a measurable subset 
$E \subset X$ satisfies the condition

$$
\operatorname{ap} \limsup _{y \rightarrow x} \frac{|f(y)-f(x)|}{d(y, x)}<\infty
$$

for a.e. $x \in E$ if and only if for any $\varepsilon>0$ there is a closed set $G \subset E$ such that $\mu(E \backslash G)<\varepsilon$ and $f$ is locally Lipschitz in $G$.

The following lemma is a variant of Lemma 3.1.5 in [7.

Lemma 4.15. Let $(X, d, \mu)$ be a complete doubling metric measure space that supports a $(1, p)$-Poincaré inequality and $V$ be a Banach space. Let $C \subset B\left(a, r_{0}\right) \subset X$, and $z \in C$ implies that $B(z, \eta) \subset B\left(a, r_{0}\right)$ and $\left.f\right|_{B(z, \eta)}$ is $M$-Lipschitz. If $a \in C$, $X \backslash C$ has density zero at $a$, and $f$ is approximately differentiable at a with respect to the strong measurable differentiable structure, then $f$ is differentiable with respect to the strong measurable differentiable structure.

Proof. Let $\left\{\left(X_{\alpha}, \varphi_{\alpha}\right)\right\}$ be the strong differentiable structure on $X$ and $a \in X_{\alpha}$. Let $\left\{\frac{\partial f}{\partial \varphi_{n}^{\alpha}}(a)\right\}$ be the "approximate differential". We write $L_{n}=\frac{\partial f}{\partial \varphi_{n}^{\alpha}}(a)$. Fix $0<\varepsilon<1$ and $0<\delta<\eta$ such that

$$
W=C \cap\left\{z:\left|f(z)-f(a)-\sum_{n=1}^{N(\alpha)}\left(\varphi_{n}^{\alpha}(z)-\varphi_{n}^{\alpha}(a)\right) L_{n}\right| \leq \varepsilon d(z, a)\right\}
$$

and $\mu(B(a, r) \backslash W)<\varepsilon \mu(B(a, r))$ whenever $0<r<\delta$.

For $x \in B\left(a,\left(1-\varepsilon^{\prime}\right) \delta\right)$, we set $r=\frac{d(x, a)}{1-\varepsilon^{\prime}}<\delta$ and observe that

$$
B\left(x, \varepsilon^{\prime} r\right) \subset B(a, r), \quad B\left(x, \varepsilon^{\prime} r\right) \cap W \neq \emptyset
$$

if we choose $\varepsilon^{\prime}$ such that $\mu\left(B\left(x, \varepsilon^{\prime} r\right)\right) \geq \varepsilon \mu(B(a, r))$. By the doubling condition of $\mu$, we may choose $\varepsilon^{\prime}=\varepsilon^{c}$, where $c$ is a positive constant depending only on $C_{\mu}$. Choose $z \in B\left(x, \varepsilon^{\prime} r\right) \cap W$ and it follows that

$$
\begin{aligned}
\left|f(x)-f(a)-\sum_{n=1}^{N(\alpha)}\left(\varphi_{n}^{\alpha}(x)-\varphi_{n}^{\alpha}(a)\right) L_{n}\right| \\
\leq\left|f(z)-f(a)-\sum_{n=1}^{N(\alpha)}\left(\varphi_{n}^{\alpha}(z)-\varphi_{n}^{\alpha}(a)\right) L_{n}\right| \\
\quad+|f(x)-f(z)|+\left|\sum_{n=1}^{N(\alpha)}\left(\varphi_{n}^{\alpha}(z)-\varphi_{n}^{\alpha}(x)\right) L_{n}\right| \\
\leq \\
\leq d(z, a)+M d(x, z)+\sum_{n=1}^{N(\alpha)}\left|L_{n}\right| \operatorname{Lip} \varphi_{n}^{\alpha} \cdot d(z, x) \\
=d(x, a) o(\varepsilon) .
\end{aligned}
$$

As a corollary of Theorem 4.14 and Lemma 4.15, we formulate the following Stepanov-type result; see also [41, Theorem 4.1].

Theorem 4.16. Let $(X, d, \mu)$ be a complete doubling metric measure space supporting a $(1, p)$-Poincaré inequality for some $p \in[1, \infty)$, and let $V$ be a Banach 
space with the Radon-Nikodym property. Then any measurable map $f: X \rightarrow V$ is differentiable at almost every point of the set

$$
\left\{x: \limsup _{y \rightarrow x} \frac{|f(y)-f(x)|}{d(y, x)}<\infty\right\}
$$

with respect to the strong measurable differentiable structure.

4.3. A general fact about the Jacobian for discrete and open mappings. Let $f: \Omega \rightarrow Y$ be a discrete, open mapping of locally bounded multiplicity such that the area formula (4.1) holds.

We point out that the Jacobian $J_{f}(x)$ can be alternately described by

$$
J_{f}(z)=\lim _{r \rightarrow 0} \frac{\mathscr{H}^{n}(f(B(z, r)))}{\mathscr{H}^{n}(B(z, r))}
$$

for a.e. $z \in \Omega$.

To see this, let $\Omega_{n}=\{x \in \Omega: N(f(x), f, \Omega)=n\}$ and $\nu_{n}=\mathscr{H}^{n}\left\llcorner_{f\left(\Omega_{n}\right)}\right.$. Then it is clear that $\Omega=\bigcup_{n=1}^{\infty} \Omega_{n}$. Let $z \in \Omega$ be a Lebesgue point of $f$. Without loss of generality, we may assume $z \in \Omega_{n}$. Then

$$
\begin{aligned}
\lim _{r \rightarrow 0} \frac{f^{*} \nu_{n}(B(z, r))}{\mathscr{H}^{n}(B(z, r))} & =\lim _{r \rightarrow 0} \frac{\nu_{n}(f(B(z, r)))}{\mathscr{H}^{n}(B(z, r))} \\
\leq & \lim _{r \rightarrow 0} \frac{\mathscr{H}^{n}\left(f\left(\Omega_{n}\right) \cap f(B(z, r))\right)}{\mathscr{H}^{n}(B(z, r))} \leq \lim _{r \rightarrow 0} \frac{\mathscr{H}^{n}(f(B(z, r)))}{\mathscr{H}^{n}(B(z, r))} .
\end{aligned}
$$

On the other hand,

$$
\mathscr{H}^{n}(f(B(z, r))) \leq f^{*} \mathscr{H}^{n}(B(z, r))=\int_{Y} N(y, f, B(z, r)) d \mathscr{H}^{n}(y)
$$

for each $r>0$. Thus

$$
\lim _{r \rightarrow 0} \frac{\mathscr{H}^{n}(f(B(z, r)))}{\mathscr{H}^{n}(B(z, r))} \leq \lim _{r \rightarrow 0} \frac{f^{*} \mathscr{H}^{n}(B(z, r))}{\mathscr{H}^{n}(B(z, r))}=\lim _{r \rightarrow 0} \frac{f^{*} \nu_{n}(B(z, r))}{\mathscr{H}^{n}(B(z, r))} .
$$

Combining (4.13) and (4.14) we obtain that

$$
J_{f}(z)=\lim _{r \rightarrow 0} \frac{\mathscr{H}^{n}(f(B(z, r)))}{\mathscr{H}^{n}(B(z, r))}
$$

for all Lebesgue points $z \in \Omega$.

Remark 4.17. The different descriptions of the Jacobian were first pointed out by Williams [4]. The first description implies that the validity of area formula (in some sense) does not depend on the differentiable structure of the space. Meanwhile, the second description reveals that the validity of the analytic description (4.12) is independent of the size of the branch set $B_{f}$.

4.4. Condition $N^{-1}$ and positiveness of the Jacobian. In this subsection, we show that $J_{f}(x)$ is positive for $\mathscr{H}^{n}$-a.e. $x \in \Omega$. The idea of the proof comes from [27. In the following proposition, $n^{\prime}=\frac{n}{n-1}$ is the conjugate exponent of $n$.

Proposition 4.18. Let $f: \Omega \rightarrow Y$ be a nonconstant mapping of finite distortion and $K(\cdot) \in L_{\text {loc }}^{n^{\prime}-1}(\Omega)$. Then $J_{f}(x)>0$ for $\mathscr{H}^{n}$-a.e. $x \in \Omega$. Moreover, $f$ satisfies Condition $N^{-1}$, i.e. for any set $E \subset \Omega$ we have

$$
\mathscr{H}^{n}(f(E))=0 \Rightarrow \mathscr{H}^{n}(E)=0 .
$$

We need the following lemma to prove Proposition 4.18 . 
Lemma 4.19. There is a constant $\tau=\tau(n)$ with the following property: For each atomless probability Borel measure $\mu$ on $Y$ there is a point $y \in Y$ and a radius $R>0$ such that

$$
\mu(B(y, 2 R)) \geq \tau \text { and } \mu(Y \backslash B(y, 3 R)) \geq \tau .
$$

Proof. Since $Y$ is Ahlfors $n$-regular, there is a constant $L$ depending only on $n$ such that any ball with radius $3 r$ can be covered by $L$ balls with radius $r$; see [35, Lemma B.4]. Set

$$
\tau=\frac{1}{L+1}
$$

Let

$$
\rho=\inf \{r>0 \text { : there exists a point } \mathrm{y} \text { in } \mathrm{Y} \text { such that } \mu(B(y, r)) \geq \tau\} .
$$

Since $\mu$ does not have atoms and $\mu(Y)<\infty$, it easily follows that $\rho>0$. We find a point $y \in Y$ and a radius $R>0$ such that

$$
\mu(B(y, 2 R)) \geq \tau \text { and } R<\rho<2 R .
$$

Consider a collection $B_{1}, \ldots, B_{L}$ of balls with radii $R$ such that they cover $B(y, 3 R)$. Since $R<\rho$, we have

$$
\mu\left(B_{i}\right) \leq \tau, i=1, \ldots, L
$$

and thus

$$
\mu(B(y, 3 R)) \leq \sum_{i=1}^{L} \mu\left(B_{i}\right) \leq L \tau,
$$

which implies, since $\mu(Y)=1$,

$$
\mu(Y \backslash B(y, 3 R)) \geq 1-L \tau=\tau .
$$

Proof of Proposition 4.18. Since the issue is local, we may assume that $f$ has bounded multiplicity in $\Omega$, i.e.

$$
N(f, \Omega) \leq N,
$$

for some positive constants $N$. We divide the proof into four steps.

Step 1 . We first prove an auxiliary estimate. Let $E \subset \Omega$ be measurable. Consider a Lipschitz function $u$ with compact support in $Y$. A direct computation gives

$$
\begin{gathered}
\int_{E} g_{u \circ f} d \mathscr{H}^{n} \leq \int_{E} g_{u} \circ f \cdot g_{f} d \mathscr{H}^{n} \leq \int_{E} g_{u} \circ f J_{f}(x)^{1 / n} K^{1 / n} d \mathscr{H}^{n}(x) \\
\leq\left(\int_{E} K^{n^{\prime}-1} d \mathscr{H}^{n}(x)\right)^{1 / n^{\prime}}\left(\int_{E}\left(g_{u} \circ f\right)^{n} J_{f}(x) d \mathscr{H}^{n}(x)\right)^{1 / n} \\
\leq\left(\int_{E} K^{n^{\prime}-1} d \mathscr{H}^{n}(x)\right)^{1 / n^{\prime}}\left(N \int_{Y} g_{u}^{n} d \mathscr{H}^{n}\right)^{1 / n} .
\end{gathered}
$$

Step 2. We claim that

$$
y_{0} \in Y \Rightarrow \mathscr{H}^{n}\left(f^{-1}\left(\left\{y_{0}\right\}\right)\right)=0 .
$$

For this, consider an arbitrary ball $B \subset \subset \Omega$ and $y_{0} \in Y$. Suppose that $f$ differs from $y_{0}$ on a set of positive measures in $B$. Then there is $R>0$ such that

$$
\kappa:=\mathscr{H}^{n}\left(B \backslash f^{-1}\left(B\left(y_{0}, R\right)\right)\right)>0 .
$$


Since singletons have zero $n$-capacity, given $\varepsilon>0$ there is a Lipschitz function $u$ on $Y$ such that

$$
\text { spt } u \in B\left(y_{0}, R\right), u\left(y_{0}\right)=1 \text { and } \int_{Y} g_{u}^{n}(x) d \mathscr{H}^{n}(x)<\varepsilon^{n} .
$$

Then

$$
\min \left\{\kappa, \mathscr{H}^{n}\left(B \cap f^{-1}\left(y_{0}\right)\right)\right\} \leq C \operatorname{diam}(B) \int_{B} g_{u \circ f} d \mathscr{H}^{n} .
$$

For this we used the well-known trick

$$
\begin{aligned}
\frac{1}{2} \min \left\{\mathscr{H}^{n}(B \cap\{v \leq 0\}), \mathscr{H}^{n}(B \cap\{v \geq 1\})\right\} \\
\leq \inf _{c \in \mathbb{R}} \int_{B}|v-c| d \mathscr{H}^{n} \leq C \operatorname{diam}(B) \int_{B} g_{v} d \mathscr{H}^{n}
\end{aligned}
$$

based on the Poincaré inequality. By (4.16), (4.19) and (4.20) we have

$$
\begin{aligned}
\min & \left\{\kappa, \mathscr{H}^{n}\left(B \cap f^{-1}\left(y_{0}\right)\right)\right\} \\
& \leq C \operatorname{diam}(B)\left(\int_{Y} g_{u}^{n} d \mathscr{H}^{n}\right)^{1 / n}\left(\int_{B} K^{n^{\prime}-1} d \mathscr{H}^{n}\right)^{1 / n^{\prime}} \\
& \leq C \operatorname{diam}(B) \varepsilon\left(\int_{B} K^{n^{\prime}-1} d \mathscr{H}^{n}\right)^{1 / n^{\prime}} .
\end{aligned}
$$

Letting $\varepsilon \rightarrow 0$ and using (4.18) we obtain that $\mathscr{H}^{n}\left(B \cap f^{-1}\left(y_{0}\right)\right)=0$ whenever $f$ differs from $y_{0}$ on a set of positive measure in $B$. Hence (4.17) follows by taking the connectedness of $\Omega$ and the assumption that $f$ is not constant into account.

Step 3. Now we prove that $J_{f}(x)>0$ for $\mathscr{H}^{n}$-a.e. $x \in \Omega$. Denote by $Z$ the zero set of the Jacobian. Fix a ball $B\left(x_{0}, r\right) \subset \Omega$. Consider the Borel measure defined by

$$
\mu(A)=\frac{\mathscr{H}^{n}\left(B\left(x_{0}, r\right) \cap f^{-1}(A)\right)}{\mathscr{H}^{n}\left(B\left(x_{0}, r\right)\right)}, A \subset Y .
$$

By (4.17), $\mu$ does not have atoms. Using Lemma 4.19 we find a point $y \in Y$ and a radius $R>0$ such that

$$
\mu(B(y, 2 R)) \geq \tau \text { and } \mu(Y \backslash B(y, 3 R)) \geq \tau .
$$

Choose a locally Lipschitz function $u$ on $Y$ such that

$$
\text { spt } u \in B(y, 3 R), u=1 \text { in } B(y, 2 R) \text { and } \int_{Y} g_{u}^{n}(x) d \mathscr{H}^{n}(x)<C(n) \text {. }
$$

Set

$$
v=u \circ f
$$

Then by (4.22) and (4.23),

$$
\begin{aligned}
& \frac{\mathscr{H}^{n}\left(B\left(x_{0}, r\right) \cap\{v=1\}\right)}{\mathscr{H}^{n}\left(B\left(x_{0}, r\right)\right)} \\
& \geq \frac{\mathscr{H}^{n}\left(B\left(x_{0}, r\right) \cap f^{-1}(B(y, 2 R))\right.}{\mathscr{H}^{n}\left(B\left(x_{0}, r\right)\right)}=\mu(B(y, 2 R)) \geq \tau,
\end{aligned}
$$


and

$$
\begin{aligned}
\frac{\mathscr{H}^{n}\left(B\left(x_{0}, r\right) \cap\{v=0\}\right)}{\mathscr{H}^{n}\left(B\left(x_{0}, r\right)\right)} \\
\quad \geq \frac{\mathscr{H}^{n}\left(B\left(x_{0}, r\right) \backslash f^{-1}(B(y, 3 R))\right.}{\mathscr{H}^{n}\left(B\left(x_{0}, r\right)\right)}=\mu(Y \backslash B(y, 3 R)) \geq \tau .
\end{aligned}
$$

By the Poincaré inequality (4.21) we have

$$
\begin{aligned}
& \quad 1 \leq \frac{C}{\mathscr{H}^{n}\left(B\left(x_{0}, r\right)\right)} \inf _{c \in \mathbb{R}} \int_{B\left(x_{0}, r\right)}|v-c| d \mathscr{H}^{n} \\
& \leq \frac{C \operatorname{diam}\left(B\left(x_{0}, r\right)\right)}{\mathscr{H}^{n}\left(B\left(x_{0}, r\right)\right)} \int_{B\left(x_{0}, r\right)} g_{v} d \mathscr{H}^{n}=\frac{C \operatorname{diam}\left(B\left(x_{0}, r\right)\right)}{\mathscr{H}^{n}\left(B\left(x_{0}, r\right)\right)} \int_{B\left(x_{0}, r\right)} g_{u \circ f} d \mathscr{H}^{n} .
\end{aligned}
$$

Since $f$ is a mapping of finite distortion, we have $g_{f}=0$ and thus $g_{u \circ f}=0, \mathscr{H}^{n}$-a.e. on $Z$. Hence by (4.26), (4.16) and (4.23),

$$
\begin{aligned}
1 \leq \frac{C \operatorname{diam}\left(B\left(x_{0}, r\right)\right)}{\mathscr{H}^{n}\left(B\left(x_{0}, r\right)\right)} \int_{B\left(x_{0}, r\right) \backslash Z} & g_{u \circ f} d \mathscr{H}^{n} \\
& \leq C\left(\operatorname{diam}\left(B\left(x_{0}, r\right)\right)^{-n} \int_{B\left(x_{0}, r\right) \backslash Z} K^{n^{\prime}-1} d \mathscr{H}^{n}\right)^{1 / n^{\prime}} .
\end{aligned}
$$

If $x_{0}$ is a Lebesgue point for $g:=K^{n^{\prime}-1} \chi_{\Omega \backslash Z}$, where $\chi_{\Omega \backslash Z}$ is the characteristic function of $\Omega \backslash Z$, it follows that $g\left(x_{0}\right)>0$. This means that the set $Z$ does not contain any Lebesgue points for $g$, and hence it must be of measure zero. Thus $J_{f}>0 \mathscr{H}^{n}$-a.e. in $\Omega$.

Step 4. We prove that $f$ satisfies Condition $N^{-1}$, i.e. $\mathscr{H}^{n}(f(E))=0$ implies $\mathscr{H}^{n}(E)=0$. Given $E \subset \Omega$ with $\mathscr{H}^{n}(f(E))=0$, we find a Borel measurable set $A \subset Y$ of measure zero which contains $f(E)$. Then $E$ is contained in the measurable set $E^{\prime}=f^{-1}(A)$. Let $h$ be the characteristic function of $A$. By the area formula we have

$$
\int_{E^{\prime}} J_{f}(x) d \mathscr{H}^{n}(x)=\int_{\Omega} h(f(x)) J_{f}(x) d \mathscr{H}^{n}(x) \leq N \int_{Y} h(y) d \mathscr{H}^{n}(y)=0 .
$$

Since $J_{f}>0 \mathscr{H}^{n}$-a.e. in $\Omega$, it follows that $\mathscr{H}^{n}(E)=0$.

Remark 4.20. In the proof of Proposition 4.18, we have implicitly used the following fact: Let $\left(f, g_{f}\right)$ be a function- minimal p-weak upper gradient pair and $u$ a Lipschitz function. Then $\left(u \circ f, g_{u} \circ f \cdot g_{f}\right)$ is a function p-weak upper gradient pair, which can be found in 15 .

\section{Generalized manifold}

From now on, all spaces are assumed to be proper (and hence locally compact), Hausdorff, connected and locally connected. $H_{c}^{*}(X)$ denotes the Alexander-Spanier cohomology groups of a space $X$ with compact supports and coefficients in $\mathbb{Z}$. From now on, $n \in \mathbb{N}$ will be a positive integer.

Definition 5.1. A space $X$ is called an $n$-dimensional, $n \geq 2$, cohomology manifold (over $\mathbb{Z}$ ), or a cohomology $n$-manifold if

(a) the cohomological dimension $\operatorname{dim}_{\mathbb{Z}} X$ is at most $n$, and 
(b) the local cohomology groups of $X$ are equivalent to $\mathbb{Z}$ in degree $n$ and to zero in degree $n-1$.

Condition (a) means that $H_{c}^{p}(U)=0$ for all open $U \subset X$ and $p \geq n+1$. Condition (b) means that for each point $x \in X$, and for each open neighborhood $U$ of $x$, there is another open neighborhood $V$ of $x$ contained in $U$ such that

$$
H_{c}^{p}(V)= \begin{cases}\mathbb{Z} & \text { if } p=n, \\ 0 & \text { if } p=n-1,\end{cases}
$$

and the standard homomorphism

$$
H_{c}^{n}(W) \rightarrow H_{c}^{n}(V)
$$

is a surjection whenever $W$ is an open neighborhood of $x$ contained in $V$. As for examples of cohomology $n$-manifolds, we point out all topological $n$-manifolds are cohomology $n$-manifolds. More examples can be found in [16].

Definition 5.2. A space $X$ is called a generalized $n$-manifold, $n \geq 2$, if it is a finite-dimensional cohomology $n$-manifold.

If a generalized $n$-manifold $X$ satisfies $H_{c}^{n}(X) \simeq \mathbb{Z}$, then $X$ is said to be orientable and a choice of a generator $g_{X}$ in $H_{c}^{n}(X)$ is called an orientation; $X$ together with $g_{X}$ is an oriented generalized $n$-manifold. If $X$ is oriented, we can simultaneously choose an orientation $g_{U}$ for all connected open subsets $U$ of $X$ via the isomorphisms

$$
H_{c}^{n}(U) \rightarrow H_{c}^{n}(U)
$$

Let $X$ and $Y$ be oriented generalized $n$-manifolds, $\Omega \subset X$ be an oriented domain and let $f: \Omega \rightarrow Y$ be continuous. For each domain $D \subset \subset \Omega$ and for each component $V$ of $Y \backslash f(\partial D)$, the map

$$
\left.f\right|_{f^{-1}(V) \cap D}: f^{-1}(V) \cap D \rightarrow V
$$

is proper. Hence we have a sequence of maps

$$
H_{c}^{n}(V) \rightarrow H_{c}^{n}\left(f^{-1}(V) \cap D\right) \rightarrow H_{c}^{n}(D),
$$

where the first map is induced by $f$ and the second map is the standard homomorphism. The composition of these two maps sends the generator $g_{V}$ to an integer multiple of the generator $g_{D}$; this integer, denoted by $\mu(y, f, D)$, is called the local degree of $f$ at a point $y \in V$ with respect to $D$. The local degree is an integer-valued locally constant function

$$
y \mapsto \mu(y, f, D)
$$

defined in $Y \backslash f(\partial D)$. If $V \cap f(D)=\emptyset$, then $\mu(y, f, D)=0$ for all $y \in V$.

From now on, we make the convention that whenever we write $f: \Omega \rightarrow Y$ is a continuous mapping between two generalized $n$-manifolds, it means that $X$ and $Y$ are two generalized $n$-manifolds and $\Omega \subset X$ is an oriented domain.

Definition 5.3. A continuous map $f: \Omega \rightarrow Y$ between two oriented generalized $n$-manifolds is said to be sense-preserving if

$$
\mu(y, f, D)>0
$$

whenever $D \subset \subset \Omega$ is a domain and $y \in f(D) \backslash f(\partial D)$.

The following properties of the local degree can be found in 16 . 
Proposition 5.4 (Basic properties of the local degree). (a) If $f, g: \Omega \rightarrow Y$ are homotopic through proper maps $h_{t}, 0 \leq t \leq 1$, such that $y \in Y \backslash h_{t}(\partial D)$ for all $t$, then

$$
\mu(y, f, D)=\mu(y, g, D) .
$$

(b) If $y \in Y \backslash h_{t}(\partial D)$ and if $f^{-1}(y) \subset D_{1} \cup \cdots \cup D_{p}$, where $D_{i}$ are all disjoint domains and contained in $D$ such that $y \in Y \backslash f\left(\partial D_{i}\right)$, then

$$
\mu(y, f, D)=\sum_{i=1}^{p} \mu\left(y, f, D_{i}\right) .
$$

(c) If $f: D \rightarrow f(D)$ is a homeomorphism, then $\mu(y, f, D)= \pm 1$ for each $y \in$ $f(D)$. In particular, if $f$ is a local homeomorphism, there is for each $x \in X$ a connected neighborhood $D$ such that $\mu(f(x), f, D)= \pm 1$. More generally, if $f$ is discrete and open and $x \in X$, then there is a relatively compact neighborhood $D$ of $x$ such that $\left\{f^{-1}(f(x))\right\} \cap \bar{D}=\{x\}$; the number $\mu(f(x), f, D)=: i(x, f)$ is independent of $D$ and called the local index of $f$ at $x$.

(d) If $f$ is open, discrete, and sense-preserving, then for each $x \in X$ there is a connected neighborhood $D$ as above such that $f(\partial D)=\partial f(D) ; D$ is called a normal neighborhood of $x$, and

$$
i(x, f)=\max _{y \in f(D)} \operatorname{card}\left\{f^{-1}(y) \cap D\right\} .
$$

If $D$ is any domain such that $f(\partial D)=\partial f(D)$, then $D$ is called a normal domain.

Definition 5.5. Let $f: \Omega \rightarrow Y$ be a continuous mapping between oriented generalized $n$-manifolds. We say that $f$ is a branched cover if $f$ is both discrete and open.

The branch set $B_{f}$ of $f: \Omega \rightarrow Y$ is the closed set of points in $\Omega$ where $f$ does not define a local homeomorphism. It was proved by Väisälä in [40] that for a branched cover $f: \Omega \rightarrow Y$ the branch set $B_{f}$ has topological dimension at most $n-2$. It follows that $\Omega \backslash B_{f}$ is connected if $f: \Omega \rightarrow Y$ is a branched cover; see [3. I, Cor 4.7].

For a branched cover $f: \Omega \rightarrow Y$ between two oriented generalized $n$-manifolds, it is either sense-preserving or sense-reversing. The latter means that $\mu(y, f, D)<0$ for each domain $D \subset \subset \Omega$ and all $y \in f(D) \backslash f(\partial D)$; see [39, Sec. 5.2].

\section{Spaces of TYPE A}

As in [16], we isolate a class of generalized manifolds whose Lipschitz analysis is similar to that on Riemannian manifolds.

Definition 6.1. Let $X$ be a metric space, and let $n \geq 2$ be an integer. Consider the following properties that $X$ may or may not possess:

(A1) $\mathrm{X}$ is $n$-rectifiable and has locally finite $\mathscr{H}^{n}$-measure;

(A2) $\mathrm{X}$ is Ahlfors $n$-regular;

(A3) $\mathrm{X}$ is locally bi-Lipschitz embeddable in Euclidean space;

(A4) $\mathrm{X}$ is locally linearly contractible.

We call $X$ a space of type $A 1, A 2, \ldots, A 12$, if it satisfies axioms $(A 1),(A 2), \ldots$, $A(1)$ and $(A 2), \ldots$ If $X$ satisfies all four axioms $(A 1)-(A 4)$, then we simply call $X$ of type $A$. Note that the dimension $n$ is fixed in the terminology.

As already mentioned before, condition $(A 1)$ means that $X$ is a countable union of Lipschitz images of subset of $\mathbb{R}^{n}$ plus a set of Hausdorff $n$-measure zero. 
Condition $(A 2)$ means that there exists a constant $C>0$ such that the Hausdorff $n$-measure in $X$ satisfies

$$
\frac{1}{C} r^{n} \leq \mathscr{H}^{n}(B(x, r)) \leq C r^{n}
$$

for all $x \in X$ and $0<r \leq \operatorname{diam}(X)$.

In the definition of $(A 3)$, the receiving Euclidean space is allowed to depend on the local embedding.

The last condition $(A 4)$ means that there exists a constant $C>0$ such that every ball $B(x, r)$ in $X$ with $0<r \leq \frac{\operatorname{diam}(X)}{C}$ is contractible in $B(x, C r)$.

We point out, for example, that every Riemannian manifold is a space of type $A$ and every Lipschitz $n$-manifold admits metrics that make the manifold into a space of type $A$. In particular, by Sullivan's theorem [38], every topological $n$-manifold, $n \neq 4$, admit type $A$ metrics. However, spaces of type $A$ need not be manifolds; see [36].

Remark 6.2. For a homology $n$-manifold $X$, the assumption $X$ is $n$-rectifiable in $(A 1)$ is indeed implied by condition $(A 2),(A 3)$ and $(A 4)$. In fact, by the work of Semmes [35], $X$ admits a $(1,1)$-Poincaré inequality. Then it follows from [4, Theorem 14.2] that $X$ is $n$-rectifiable; see also [11, Proposition 2.1].

As pointed out by Heinonen and Rickmann [16], spaces of type $A$ will allow for differential calculus akin to that in Euclidean spaces. This follows from the work of Semmes [35] which shows that (locally) a space of type $A$ admits a (1,1)-Poincaré type inequality in the sense of Section 2, Properties $(A 1)$ and $(A 2)$ guarantee that the usual Lipschitz calculus on rectifiable sets is at our disposal.

Assume that $X$ is an oriented generalized $n$-manifold, and assume that $X$ is of type $A 123$. Let $U$ be an open connected neighborhood of a point in $X$ that can be embedded bi-Lipschitz in some $\mathbb{R}^{N}$ and that $U$ has finite Hausdorff $n$-measure. Because of properties $(A 1)$ and $(A 2)$, the set $U$ has a tangent $n$-plane $T_{x} U$ at a.e. point $x \in U$. The collection of these planes is called a measurable tangent bundle of $U$, and it is denoted by $T U$.

Each $n$-plane $T_{x} U$, whenever it exists, is an $n$-dimensional subspace of $\mathbb{R}^{N}$, and a measurable choice of orientations $\xi=\left(\xi_{x}\right)$ on each $T_{x} U$ is called an orientation of the tangent bundle $T U$. Such orientations always exist. Because $X$ is an oriented generalized $n$-manifold, there is another orientation on $U$, provided $U$ is connected; this is a generator $g_{U}$ in the group $H_{c}^{n}(U)=\mathbb{Z}$ determined by the fixed orientation on $X$.

Fix a point $x \in U$ such that $T_{x} U$ exists. Then the projection

$$
\pi_{x}: \mathbb{R}^{N} \rightarrow T_{x} U+x
$$

satisfies $x \notin \pi_{x}(\partial D)$ whenever $D$ is a sufficiently small open connected neighborhood of $x$ in $U$. To see it, consider the set

$$
\Lambda=\left\{y \in \mathbb{R}^{N}: \operatorname{dist}\left(y-x, T_{x} U\right)>\varepsilon|y-x|\right\} .
$$

We claim for sufficiently small open connected neighborhood $D$ of $x$, it must hold that $\Lambda \cap D=\emptyset$ for each $\varepsilon>0$. If not, the $n$-regularity condition $(A 2)$ implies that $U$ has positive $n$-density along the set $\Lambda$, contradicting the definition for approximate tangent planes; see [7, Theorem 3.2.19]. Moreover, one can easily show that for any 
$\varepsilon>0$

$$
\frac{\left|x-x_{0}\right|}{2} \leq \pi_{x_{0}}\left(x-x_{0}\right) \leq \varepsilon\left|x-x_{0}\right|
$$

whenever $\left|x-x_{0}\right|$ is small enough; see [7, Section 3.2.16]. Thus, if $V$ is the $x$ component of $\left(T_{x} U+x\right) \backslash \pi_{x}(\partial D)$, we have

$$
H_{c}^{n}\left(T_{x} U\right) \leftarrow H_{c}^{n}(V) \stackrel{\pi_{x}^{*}}{\rightarrow} H_{c}^{n}\left(\pi_{x}^{-1}(V) \cap D\right) \rightarrow H_{c}^{n}(D) \rightarrow H_{c}^{n}(U),
$$

where the unnamed arrows represent a canonical isomorphism. If the orientations $\xi_{U}$ and $g_{U}$ correspond to each other under the map in (6.4), we say that $T_{x} U$ and $U$ are coherently oriented at $x$ by $\xi_{x}$ and $g_{U}$; if a measurable coherent orientation $\xi=\left(\xi_{x}\right)$ is chosen at almost every point, we say that $T U$ is metrically oriented by $\xi$ and $g_{U}$.

Proposition 6.3 ([17], Exam.3.10). Every generalized $n$-manifold of type $A$ can be (locally) metrically oriented.

\section{SIZE OF THE BRANCH SET}

From now on, mappings of finite distortion are assumed to be sense-preserving. The fact that both $B_{f}$ and $f\left(B_{f}\right)$ has measure zero is of great importance in the theory of mappings of finite distortion in Euclidean space. In this subsection, we show that, under suitable integrability condition for the distortion function for the map $f$, both $B_{f}$ and $f\left(B_{f}\right)$ has zero $n$-Hausdorff measure for a mapping $f$ of finite distortion between generalized $n$-manifolds of type $A$.

Before we get to the statement of the main result of this section, let us recall that a mapping $f$ of an $n$-rectifiable subset $\Omega$ of $\mathbb{R}^{N}$ into $\mathbb{R}^{M}$ is approximately differentiable at $x_{0} \in \Omega$ if $x_{0}$ has a neighborhood in $\Omega$ of finite $\mathscr{H}^{n}$-measure and if there exists $y_{0} \in \mathbb{R}^{M}$ and a linear map $T: \mathbb{R}^{N} \rightarrow \mathbb{R}^{M}$ such that

$$
\lim _{r \rightarrow 0} \mathscr{H}^{n}\left(\left\{x:\left|x-x_{0}\right|<r \text { and }\left|f(x)-y_{0}-T\left(x-x_{0}\right)\right| \geq \varepsilon\left|x-x_{0}\right|\right\}\right) r^{-n}=0
$$

for each $\varepsilon>0$. Equivalently, if there exist a neighborhood $\mathrm{U}$ of $x_{0}$ in $\mathbb{R}^{N}$ and a mapping $g: U \rightarrow \mathbb{R}^{M}$, which is differentiable at $a$, such that

$$
\limsup _{r \rightarrow 0} \frac{\mathscr{H}^{n}\left(\{x: f(x) \neq g(x)\} \cap B\left(x_{0}, r\right)\right)}{r^{n}}=0 .
$$

If a tangent $n$-plane $T_{x_{0}} \Omega$ exists at $x_{0}$, the restriction of $T$ to $T_{x_{0}} \Omega$ is the approximate differential of $f$ at $x_{0}$, and it is denoted by ap- $D f\left(x_{0}\right)$. The approximately differential ap- $D f\left(x_{0}\right)$ is unique; see [16, Section 6.5] or [24, Remark 2.13].

Theorem 7.1. Let $f: \Omega \rightarrow Y$ be a mapping of finite distortion with $K(\cdot) \in$ $L_{l o c}^{n^{\prime}-1}(\Omega)$. Then it holds that $\mathscr{H}^{n}\left(B_{f}\right)=\mathscr{H}^{n}\left(f\left(B_{f}\right)\right)=0$.

Proof. Since $f$ satisfies condition $N$, it suffices to show that $\mathscr{H}^{n}\left(B_{f}\right)=0$. Because the issue is local, and the conclusion is bi-Lipschitz invariant, we may assume that $\Omega$ and $Y$ are metrically oriented subsets of some Euclidean space. In this framework, the claim follows from Proposition 7.2 and Proposition 7.3 below.

Proposition 7.2. Let $\Omega$ and $Y$ be metrically oriented subsets of some Euclidean spaces and $f: \Omega \rightarrow Y$ a mapping of finite distortion. Then for $\mathscr{H}^{n}$-a.e. $x_{0} \in \Omega$, 
there exists a linear map $T: \mathbb{R}^{N} \rightarrow \mathbb{R}^{M}$ such that

$$
\lim _{x \rightarrow x_{0}, x \in \Omega} \frac{\left|f(x)-f\left(x_{0}\right)-T\left(x-x_{0}\right)\right|}{\left|x-x_{0}\right|}=0 .
$$

Proof. We first note that $f$ is approximately differentiable $\mathscr{H}^{n}$-a.e. To see it, let $\Omega=\left(\bigcup_{k=1}^{\infty} A_{k}\right) \cup E$ be as in Lemma 4.3. Then $\left.f\right|_{A_{k}}$ is $k$-Lipschitz. By [7, Theorem 3.2.19], $\left.f\right|_{A_{k}}$ is approximately differentiable at $\mathscr{H}^{n}$-almost every point in $E_{k}$. Since every point in $A_{k}$ is a point of $\mathscr{H}^{n}$ density, we conclude that $f$ is approximately differentiable $\mathscr{H}^{n}$-a.e. in $\Omega$. Note also that if the approximately differentiable point is such that the tangent $n$-plane $T_{x_{0}} \Omega$ exists, then the restriction of $T$ to $T_{x_{0}} \Omega$ is unique (by the Ahlfors $n$-regularity). On the other hand, Theorem 8.1 in [1] implies that $(\Omega, \mathbf{x})$ is a strong measurable differentiable structure on $\left(\Omega,|\cdot|, \mathscr{H}^{n}\right)$, where $\mathbf{x}: \Omega \rightarrow \mathbb{R}^{N}$ is defined by $\mathbf{x}(x)=x$. Theorem 4.16 and Proposition 4.6 then allow us to complete the proof.

If (7.1) holds at $x_{0} \in \Omega$ and the tangent $n$-plane $T_{x_{0}} \Omega$ exists, we denote by $D f\left(x_{0}\right)$ the restriction of $T$ to $T_{x_{0}} \Omega$ and call it a differentiable point of $f$. In this sense, Proposition 7.2 implies that $f$ is a differentiable at $\mathscr{H}^{n}$-a.e. points in $\Omega$.

Next assume that $\Omega$ and $Y$ are two metrically oriented generalized $n$-manifolds of type $A$ lying in some Euclidean space. Denote the respective orientations by $\left(\xi, g_{\Omega}\right)$ and $\left(\eta, g_{Y}\right)$. Let $O T_{\Omega}$ be the set of all points in $\Omega$ such that $T_{x} \Omega$ exists, that $T_{x} \Omega$ and $\Omega$ are coherently oriented by $\xi_{x}$ and $g_{\Omega}$, and that $\Omega$ has Hausdorff $n$-measure density 1 at $x$; see [7, Theorem 3.2.22]. The set $O T_{\Omega}$ has full measure in $\Omega$. We denote $O T_{Y}$ similarly.

If $f: \Omega \rightarrow Y$ is as in Theorem 17.1, it follows from Proposition 4.18 and Corollary 4.2 that the sets $f^{-1}\left(O T_{Y}\right) \cap O T_{\Omega}$ and $f\left(O T_{\Omega}\right) \cap O T_{Y}$ have full measure in $\Omega$ and $f(\Omega)$, respectively. We also denote by $P_{f}$ the set of points in $\Omega$ where the Jacobian is positive. It follows from Proposition 4.18 that $P_{f}$ have full measure in $\Omega$.

Proposition 7.3. Let $f: \Omega \rightarrow Y$ be as in Theorem 17.1, with an additional assumption that $\Omega$ and $Y$ are metrically oriented subsets of some Euclidean space. Then $f$ cannot be differentiable at any point in $B_{f} \cap f^{-1}\left(O T_{Y}\right) \cap O T_{\Omega} \cap P_{f}$.

Proof. Let $x_{0} \in f^{-1}\left(O T_{Y}\right) \cap O T_{\Omega} \cap P_{f}$ be a point where $f$ is differentiable and $J_{f}\left(x_{0}\right)>0$. Then the differential $D f\left(x_{0}\right)$ cannot be degenerate; that is, it has to map the tangent plane $T_{x_{0}} \Omega$ onto the tangent plane $T_{y_{0}} Y$, where $f\left(x_{0}\right)=y_{0}$; see [7, Theorem 3.2.22].

Moreover, we claim that for a sufficiently small neighborhood $D:=B\left(x_{0}, r\right)$ of $x_{0}$ in $\Omega$, the maps $\overline{D f}\left(x_{0}\right) \circ \pi_{x_{0}}$ and $\pi_{y_{0}} \circ f$ are homotopic as maps $D \rightarrow T_{y_{0}} Y+y_{0}$ and $y_{0}$ has a positive distance away from the image of $\partial D$ under the homotopy, where $\pi_{*}$ denotes the projection map, and $\overline{D f}\left(x_{0}\right)$ is the affine map between the planes $T_{x_{0}} \Omega+x_{0}$ and $T_{y_{0}} Y+y_{0}$ determined by the differential $D f\left(x_{0}\right)$. In fact, we may use the straight-line homotopy, i.e. $H_{t}(x)=(1-t) \overline{D f}\left(x_{0}\right) \circ \pi_{x_{0}}(x)+t \pi_{y_{0}} \circ f(x)$. Now we only need to show that $y_{0} \notin H_{t}(\partial D)$ for all $t \in[0,1]$. To this end, set

$$
\lambda=\inf _{|x|=1}\left|\overline{D f}\left(x_{0}\right) x\right| .
$$

Since $J_{f}\left(x_{0}\right)>0, \lambda>0$, it follows that

$$
\left|\overline{D f}\left(x_{0}\right) \pi_{x_{0}}\left(x-x_{0}\right)\right| \geq \frac{\lambda}{2}\left|\pi_{x_{0}}\left(x-x_{0}\right)\right| \geq \frac{\lambda}{4}\left|x-x_{0}\right|,
$$


when $\left|x-x_{0}\right|$ are small enough. Then for any $x \in \partial D$,

$$
\begin{aligned}
\left|H_{t}(x)-y_{0}\right| & =\left|(1-t) \overline{D f}\left(x_{0}\right) \circ \pi_{x_{0}}(x)+t \pi_{y_{0}} \circ f(x)-y_{0}\right| \\
& \geq\left|\overline{D f}\left(x_{0}\right) \circ \pi_{x_{0}}(x)-y_{0}\right|-t\left|\pi_{y_{0}} \circ f(x)-\overline{D f}\left(x_{0}\right) \circ \pi_{x_{0}}(x)\right| \\
& =\left|\overline{D f}\left(x_{0}\right) \circ \pi_{x_{0}}\left(x-x_{0}\right)\right|-t\left|\pi_{y_{0}} \circ f(x)-\overline{D f}\left(x_{0}\right) \circ \pi_{x_{0}}(x)\right| \\
& \geq \frac{\lambda}{4}\left|x-x_{0}\right|-t\left|\pi_{y_{0}} \circ f(x)-\overline{D f}\left(x_{0}\right) \circ \pi_{x_{0}}(x)\right| .
\end{aligned}
$$

We next estimate the second term,

$$
\begin{aligned}
& \left|\pi_{y_{0}} \circ f(x)-\overline{D f}\left(x_{0}\right) \circ \pi_{x_{0}}(x)\right|=\left|\pi_{y_{0}} \circ\left(f(x)-y_{0}\right)-\overline{D f}\left(x_{0}\right) \circ \pi_{x_{0}}\left(x-x_{0}\right)\right| \\
& \leq\left|\pi_{y_{0}}\left(f(x)-y_{0}\right)-\left(f(x)-y_{0}\right)\right|+\left|f(x)-f\left(x_{0}\right)-\overline{D f}\left(x_{0}\right)\left(x-x_{0}\right)\right| \\
& \quad+\left|\overline{D f}\left(x_{0}\right) \circ \pi_{x_{0}}\left(x-x_{0}\right)-\overline{D f}\left(x_{0}\right)\left(x-x_{0}\right)\right|:=I_{1}+I_{2}+I_{3} .
\end{aligned}
$$

The differentiability of $f$ at $x_{0}$ implies that

$$
I_{1} \leq\left|D f\left(x_{0}\right)\right| o\left(\left|x-x_{0}\right|\right)
$$

and

$$
I_{2} \leq\left|x-x_{0}\right| o\left(\left|x-x_{0}\right|\right) .
$$

The property of $\pi_{*}$ easily implies

$$
I_{3} \leq\left|D f\left(x_{0}\right)\right| o\left(\left|x-x_{0}\right|\right)
$$

Combining the estimates (7.2) with (7.3), (7.4) and (7.5) gives

$$
\left|H_{t}(x)-y_{0}\right| \geq \frac{\lambda}{5}\left|x-x_{0}\right|
$$

when $\left|x-x_{0}\right|$ is sufficiently small. Thus $y_{0} \notin H_{t}(\partial D)$ for all $t \in[0,1]$ if $D$ is sufficiently small.

By using this and the definition for coherent orientation, it is clear that $f$ must have local index $i\left(x_{0}, f\right)=1$ at $x_{0}$. In particular, $f$ is a local homeomorphism at $x_{0}$, and the proposition follows.

Remark 7.4. i) The assumption that $f$ is sense-preserving in Proposition 7.3 is crucial; see [16, Remark 6.7(a)].

ii) Theorem 7.1 is sharp in the following sense: for each $n \geq 3$ and $\varepsilon>0$ there is a quasiregular map $f: \mathbb{R}^{n} \rightarrow \mathbb{R}^{n}$ such that the Hausdorff dimension of $B_{f}$ is at least $n-\varepsilon$; see [16, Remark 6.7(b)].

\section{ACKNOWLEDGEMENTS}

The author wishes to thank his supervisor Academy Professor Pekka Koskela and Professor Kai Rajala for numerous suggestions. He is also grateful to Marshall Williams, Ville Kirsilä and Thomas Zürcher for many useful discussions on related topics. Finally, he would like to thank the referee for numerous pointed comments that improve on the exposition. 


\section{REFERENCES}

[1] L. Ambrosio and B. Kirchheim, Rectifiable sets in metric and Banach spaces, Math. Ann. 318 (2000), no. 3, 527-555, DOI 10.1007/s002080000122. MR1800768 (2003a:28009)

[2] A. Björn and J. Björn, Nonlinear potential theory on metric spaces, EMS Tracts in Mathematics, vol. 17, European Mathematical Society (EMS), Zürich, 2011. MR2867756

[3] A. Borel, Seminar on transformation groups, With contributions by G. Bredon, E. E. Floyd, D. Montgomery, R. Palais. Annals of Mathematics Studies, No. 46, Princeton University Press, Princeton, N.J., 1960. MR0116341 (22 \#7129)

[4] J. Cheeger, Differentiability of Lipschitz functions on metric measure spaces, Geom. Funct. Anal. 9 (1999), no. 3, 428-517, DOI 10.1007/s000390050094. MR:1708448 (2000g:53043)

[5] J. Cheeger and B. Kleiner, Differentiability of Lipschitz maps from metric measure spaces to Banach spaces with the Radon-Nikodým property, Geom. Funct. Anal. 19 (2009), no. 4, 1017-1028, DOI 10.1007/s00039-009-0030-6. MR2570313 (2011c:30138)

[6] E. Durand-Cartagena, L. Ihnatsyeva, R. Korte, and M. Szumańska, On Whitney-type characterization of approximate differentiability on metric measure spaces, Canad. J. Math. 66 (2014), no. 4, 721-742, DOI 10.4153/CJM-2012-064-x. MR3224261

[7] H. Federer, Geometric measure theory, Die Grundlehren der mathematischen Wissenschaften, Band 153, Springer-Verlag New York Inc., New York, 1969. MR0257325(41 \#1976)

[8] P. Hajłasz and P. Koskela, Sobolev met Poincaré, Mem. Amer. Math. Soc. 145 (2000), no. 688, $\mathrm{x}+101$, DOI 10.1090/memo/0688. MR1683160 (2000j:46063)

[9] J. Heinonen, Lectures on analysis on metric spaces, Universitext, Springer-Verlag, New York, 2001. MR1800917 (2002c:30028)

[10] J. Heinonen, Nonsmooth calculus, Bull. Amer. Math. Soc. (N.S.) 44 (2007), no. 2, 163-232, DOI 10.1090/S0273-0979-07-01140-8. MR2291675 (2008e:49021)

[11] J. Heinonen and S. Keith, Flat forms, bi-Lipschitz parameterizations, and smoothability of manifolds, Publ. Math. Inst. Hautes Études Sci. 113 (2011), 1-37, DOI 10.1007/s10240-0110032-4. MR2805596 (2012h:30194)

[12] J. Heinonen and P. Koskela, Quasiconformal maps in metric spaces with controlled geometry, Acta Math. 181 (1998), no. 1, 1-61, DOI 10.1007/BF02392747. MR1654771 (99j:30025)

[13] J. Heinonen and P. Koskela, A note on Lipschitz functions, upper gradients, and the Poincaré inequality, New Zealand J. Math. 28 (1999), no. 1, 37-42. MR1691958(2000d:46041)

[14] J. Heinonen, P. Koskela, N. Shanmugalingam, and J. T. Tyson, Sobolev classes of Banach space-valued functions and quasiconformal mappings, J. Anal. Math. 85 (2001), 87-139, DOI 10.1007/BF02788076. MR.1869604 (2002k:46090)

[15] J. Heinonen, P. Koskela, N. Shanmugalingam and J. T. Tyson, Sobolev spaces on metric measure spaces: an approach based on upper gradients, Cambridge Studies in Advanced Mathematics Series 2015 (To appear).

[16] J. Heinonen and S. Rickman, Geometric branched covers between generalized manifolds, Duke Math. J. 113 (2002), no. 3, 465-529, DOI 10.1215/S0012-7094-02-11333-7. MR.1909607 (2003h:57003)

[17] J. Heinonen and D. Sullivan, On the locally branched Euclidean metric gauge, Duke Math. J. 114 (2002), no. 1, 15-41, DOI 10.1215/S0012-7094-02-11412-4. MR1915034 (2004b:30044)

[18] S. Hencl and P. Koskela, Lectures on mappings of finite distortion, Lecture Notes in Mathematics, vol. 2096, Springer, Cham, 2014. MR.3184742

[19] J. G. Hocking and G. S. Young, Topology, 2nd ed., Dover Publications, Inc., New York, 1988. MR.1016814(90h:54001)

[20] T. Iwaniec, P. Koskela, and J. Onninen, Mappings of finite distortion: monotonicity and continuity, Invent. Math. 144 (2001), no. 3, 507-531, DOI 10.1007/s002220100130. MR1833892 (2002c:30029)

[21] T. Iwaniec and G. Martin, Geometric function theory and non-linear analysis, Oxford Mathematical Monographs, The Clarendon Press, Oxford University Press, New York, 2001. MR.1859913(2003c:30001)

[22] J. Kauhanen, P. Koskela, and J. Malý, Mappings of finite distortion: discreteness and openness, Arch. Ration. Mech. Anal. 160 (2001), no. 2, 135-151, DOI 10.1007/s002050100162. MR.1864838 (2002m:30023) 
[23] J. Kauhanen, P. Koskela, and J. Malý, Mappings of finite distortion: condition N, Michigan Math. J. 49 (2001), no. 1, 169-181, DOI 10.1307/mmj/1008719040. MR 1827080 (2002d:30027)

[24] S. Keith, Measurable differentiable structures and the Poincaré inequality, Indiana Univ. Math. J. 53 (2004), no. 4, 1127-1150, DOI 10.1512/iumj.2004.53.2417. MR2095451 (2005g:53068)

[25] V. Kirsilä, Mappings of finite distortion from generalized manifolds, Conform. Geom. Dyn. 18 (2014), 229-262, DOI 10.1090/S1088-4173-2014-00272-0. MR.3278159

[26] P. Koskela and P. MacManus, Quasiconformal mappings and Sobolev spaces, Studia Math. 131 (1998), no. 1, 1-17. MR1628655(99e:46042)

[27] P. Koskela and J. Malý, Mappings of finite distortion: the zero set of the Jacobian, J. Eur. Math. Soc. (JEMS) 5 (2003), no. 2, 95-105, DOI 10.1007/s10097-002-0046-9. MR1985612 (2005d:30031)

[28] V. Magnani, An area formula in metric spaces, Colloq. Math. 124 (2011), no. 2, 275-283, DOI 10.4064/cm124-2-11. MR2842953

[29] O. Martio, S. Rickman, and J. Väisälä, Definitions for quasiregular mappings, Ann. Acad. Sci. Fenn. Ser. A I No. 448 (1969), 40. MR.0259114 (41 \#3756)

[30] O. Martio, S. Rickman, and J. Väisälä, Distortion and singularities of quasiregular mappings, Ann. Acad. Sci. Fenn. Ser. A I No. 465 (1970), 13. MR0267093 (42 \#1995)

[31] O. Martio, S. Rickman, and J. Väisälä, Topological and metric properties of quasiregular mappings, Ann. Acad. Sci. Fenn. Ser. A I 488 (1971), 31. MR0299782 (45 \#8830)

[32] J. Onninen and K. Rajala, Quasiregular mappings to generalized manifolds, J. Anal. Math. 109 (2009), 33-79, DOI 10.1007/s11854-009-0028-x. MR2585391(2011c:30063)

[33] Yu. G. Reshetnyak, Space mappings with bounded distortion, Translations of Mathematical Monographs, vol. 73, American Mathematical Society, Providence, RI, 1989. Translated from the Russian by H. H. McFaden. MR994644 (90d:30067)

[34] S. Rickman, Quasiregular mappings, Ergebnisse der Mathematik und ihrer Grenzgebiete (3) [Results in Mathematics and Related Areas (3)], vol. 26, Springer-Verlag, Berlin, 1993. MR:1238941 (95g:30026)

[35] S. Semmes, Finding curves on general spaces through quantitative topology, with applications to Sobolev and Poincaré inequalities, Selecta Math. (N.S.) 2 (1996), no. 2, 155-295, DOI 10.1007/BF01587936. MR1414889 (97j:46033)

[36] S. Semmes, Good metric spaces without good parameterizations, Rev. Mat. Iberoamericana 12 (1996), no. 1, 187-275, DOI 10.4171/RMI/198. MR1387590(97e:57025)

[37] N. Shanmugalingam, Newtonian spaces: an extension of Sobolev spaces to metric measure spaces, Rev. Mat. Iberoamericana 16 (2000), no. 2, 243-279, DOI 10.4171/RMI/275. MR:1809341 (2002b:46059)

[38] D. Sullivan, Hyperbolic geometry and homeomorphisms, Geometric topology (Proc. Georgia Topology Conf., Athens, Ga., 1977), Academic Press, New York-London, 1979, pp. 543-555. MR.537749 (81m:57012)

[39] J. Väisälä, Minimal mappings in euclidean spaces, Ann. Acad. Sci. Fenn. Ser. A I No. 366 (1965), 22. MR0178454(31 \#2711)

[40] J. Väisälä, Discrete open mappings on manifolds, Ann. Acad. Sci. Fenn. Ser. A I No. 392 (1966), 10. MR0200928 (34 \#814)

[41] K. Wildrick and T. Zürcher, Sharp differentiability results for the lower local Lipschitz constant and applications to non-embedding, J. Geom. Anal. (to appear).

[42] M. Williams, Geometric and analytic quasiconformality in metric measure spaces, Proc. Amer. Math. Soc. 140 (2012), no. 4, 1251-1266, DOI 10.1090/S0002-9939-2011-11035-9. MR:2869110(2012m:30063)

[43] M. Williams, Dilatation, pointwise Lipschitz constants, and condition $N$ on curves, Michigan Math. J. 63 (2014), no. 4, 687-700, DOI 10.1307/mmj/1417799221. MR3286666

[44] M. Williams, Definitions of quasiregularity in metric measure spaces, preprint 2015.

Department of Mathematics and Statistics, University of Jyväskylä, P.O. Box 35, FI-40014 UNIVERSity OF JYVÄSKYlä, FinlAND

E-mail address: changyu.c.guo@jyu.fi 